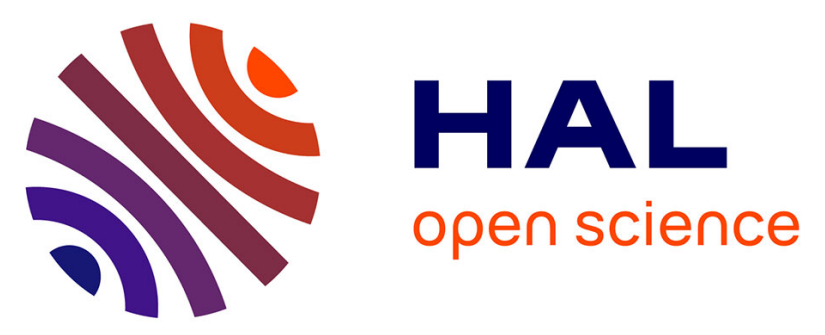

\title{
RAD50 promotes DNA repair by homologous recombination and restrains antigenic variation in African trypanosomes
}

Ann-Kathrin Mehnert, Marco Prorocic, Annick Dujeancourt-Henry, Sebastian Hutchinson, Richard Mcculloch, Lucy Glover

\section{To cite this version:}

Ann-Kathrin Mehnert, Marco Prorocic, Annick Dujeancourt-Henry, Sebastian Hutchinson, Richard Mcculloch, et al.. RAD50 promotes DNA repair by homologous recombination and restrains antigenic variation in African trypanosomes. 2021. pasteur-03107143

\section{HAL Id: pasteur-03107143}

https://hal-pasteur.archives-ouvertes.fr/pasteur-03107143

Preprint submitted on 12 Jan 2021

HAL is a multi-disciplinary open access archive for the deposit and dissemination of scientific research documents, whether they are published or not. The documents may come from teaching and research institutions in France or abroad, or from public or private research centers.
L'archive ouverte pluridisciplinaire HAL, est destinée au dépôt et à la diffusion de documents scientifiques de niveau recherche, publiés ou non, émanant des établissements d'enseignement et de recherche français ou étrangers, des laboratoires publics ou privés.

$$
\text { Copyright }
$$




\section{RAD50 promotes DNA repair by homologous recombination and}

2 restrains antigenic variation in African trypanosomes

3

Ann-Kathrin Mehnert ${ }^{1}$, Marco Prorocic ${ }^{2}$, Annick Dujeancourt-Henry ${ }^{1}$, Sebastian Hutchinson ${ }^{3}$, Richard McCulloch $^{2}$, Lucy Glover ${ }^{1 *}$

1 Trypanosome Molecular Biology, Department of Parasites and Insect Vectors, Institut Pasteur, 25-28 Rue du Docteur Roux 75015, Paris, France.

${ }^{2}$ Wellcome Center for Integrative Parasitology, Sir Graeme Davis Building, 120 University Place, Glasgow, G12 8TA, UK.

${ }^{3}$ Trypanosome Cell Biology, Department of Parasites and Insect Vectors, Institut Pasteur, 25-28 Rue du Docteur Roux 75015, Paris, France.

Present address: : Centre for Infectious Diseases, Virology, Heidelberg University Hospital, Im Neuenheimer Feld 344, 69120 Heidelberg, Germany

* To whom correspondence should be addressed lucy.glover@pasteur.fr; Tel: +33 140613425

KEYWORDS: VSG, RAD50, DNA damage.

Abstract 147 words

\section{ABSTRACT}

Homologous recombination dominates as the major form of DNA repair in Trypanosoma brucei, and is especially important for recombination of the subtelomeric variant surface glycoprotein during antigenic variation. RAD50, a component of the MRN complex (MRE11, RAD50, NBS1), is central to homologous recombination through facilitating resection and governing the DNA damage response. The function of RAD50 in trypanosomes is untested. Here we report that RAD50 is required for RAD51-dependent homologous recombination, phosphorylation of histone $\mathrm{H} 2 \mathrm{~A}$ and controlled resection following a DNA double strand break (DSB). Perhaps surprisingly, DSB resection in the rad50 nulls was not impaired and appeared to peak earlier than in the parental strains. Finally, we show that RAD50 suppresses DNA repair using donors with short stretches of homology at a subtelomeric locus, with null strains producing a greater diversity of expressed VSG variants following DSB repair. We conclude that RAD50 promotes stringent homologous recombination at subtelomeric loci and restrains antigenic variation. 


\section{INTRODUCTION}

Trypanosoma brucei (T. brucei) is a protozoan parasite and the causative agent of human African trypanosomiasis, or sleeping sickness, and nagana in cattle. Trypanosomes cycle between their insect vector, the tsetse fly, and mammalian hosts, where they colonise the blood, fat ${ }^{1}$ and skin $^{2}$ and eventually cross the blood brain barrier in late stage infection. If left untreated, trypanosomiasis is normally fatal ${ }^{3}$. In the mammalian host, each trypanosome cell is covered in a dense layer of a single species of variant surface glycoprotein (VSG). The highly immunogenic VSG layer ${ }^{4,5}$ acts as an barrier, concealing other surface components from the host immune response ${ }^{6}$. Trypanosomes maintain a persistent infection by continuously escaping the host's immune response though antigenic variation ${ }^{7}$. Central to this survival strategy is monoallelic expression of the VSG from a subtelomeric locus, known as an expression site (VSG-ES), and stochastic VSG switching. The $\sim 15$ VSG-ESs in the trypanosome genome share a high degree of sequence and structure conservation ${ }^{8}$, each being an RNA polymerase-I (RNA Pol-I) polycistronic transcription unit with a single VSG gene found adjacent to the telomere, up to $60 \mathrm{~kb}$ downstream of the promoter ${ }^{8}$. The VSG gene is flanked by two sets of repetitive sequence: downstream is the telomere, and upstream is a block of repetitive sequence, known as the 70-bp repeats, which modulates VSG switching ${ }^{8,9}$. Characteristic of a trypanosome infection are recrudescent waves of parasitemia, each of which is composed of a diverse VSG expressing population, with between 7 - 79 VSGs detected in each peak of parasitemia ${ }^{10-12}$. VSG diversity arises through altering the single VSG-ES that is transcribed or, more commonly, by recombination of silent VSGs into the active VSG-ES. The seemingly unrestricted use of VSG genes might be expected to result in a rapid exhaustion of the VSG gene repertoire. However, the parasite's ability to sustain an infection appears to lie in an enormous repertoire of $>2000$ VSG genes and pseudogenes ${ }^{13-15}$, mainly found in subtelomeric VSG arrays, and a capacity for generation of novel 'mosaic' VSG genes through segmental gene conversion of multiple (pseudo) VSGs, in particular late in infection ${ }^{10,11,14}$. Importantly, almost all of the array VSGs are associated with upstream tracts of 70bp repeats, providing the necessary substrate needed for homologous recombination mediated antigenic variation ${ }^{16}$.

A DNA double-strand break (DSB) is an extremely toxic lesion in any cell, which if left unrepaired can lead to cell death. In T. brucei RAD51-dependent homologous recombination (HR) dominates as the major DNA repair and recombination pathway, with microhomology mediated end-joining (MMEJ) playing a minor role ${ }^{17-20}$. HR is important for VSG switching, and though it is not clear how MMEJ acts in this reaction, repair of induced DSBs can occur by coupled HR and MMEJ, and MMEJ is more frequently used for repair of DSBs induced within the VSG-ES ${ }^{21}$. Unrepaired DSBs appear to persist throughout the cell cycle without inhibiting the trypanosomes ability to replicate their DNA ${ }^{22}$, but whether HR or MMEJ are regulated is unknown. In addition, non-homologous end-joining (NHEJ) appears to be absent in trypanosomes ${ }^{21,23,24}$. These features of trypanosome DSB repair contrast with mammalian cells, where NHEJ is highly active, $\mathrm{HR}$ predominates in $\mathrm{S}$ and $\mathrm{G}_{2}$ phase cells and MMEJ is considered a minor reaction ${ }^{25}$. In trypanosomes both transcriptionally active and silent 
subtelomeres are fragile ${ }^{26,27}$, and accumulate natural breaks. Within the active VSG-ES specifically, a DSB between the VSG and 70-bp repeats acts as a potent driver of antigenic variation and precipitates VSG switching ${ }^{27}$. Several DNA repair and recombination proteins have been shown to be important for antigenic variation in trypanosomes, thus linking VSG switching with this process: loss of RAD51 ${ }^{18}$, the RAD51-3 paralogue ${ }^{28}$, or the RAD51-interacting protein BRCA2 ${ }^{29,30}$ results in impaired VSG switching, while loss of RECQ2 ${ }^{31}$, TOPO3 $\alpha$ or RMI1 increases VSG switching ${ }^{32,33}$, as does loss of the histone variants H3.V and H4.V ${ }^{15}$. Loss of ATR, which is involved in DNA damage signalling, impairs monoallelic VSG expression and increases VSG switching through localized DNA damage ${ }^{34}$. Histone Acetyltransferase (HAT3) is required for recombination repair of a chromosomeinternal DSB, but suppresses DSB repair within the VSG-ES which suggests repair is compartmentalised in trypanosomes ${ }^{35}$.

The DNA damage response (DDR) is an orchestrated cellular response to many different genome lesions, including DSBs, which most commonly form via stalled replication forks ${ }^{36}$. Critical to DSB repair is the MRE11 - RAD50 - NBS1 (MRN) complex (in yeast MRE11 - RAD50 - XRS1, MRX), which acts as a DNA damage sensing complex and is responsible for recognizing the free DNA ends, where it is one of the first complexes to bind and initiate HR ${ }^{37,38}$. MRE11-RAD50 forms the core of this complex and is conserved across all domains of life, whereas NBS1 only forms part of the complex in eukaryotes ${ }^{37}$. MRN consists of two molecules of each component protein, and diffuses along homoduplex DNA searching for free DNA ends - a process that is driven by RAD50 ${ }^{39}$. The MRE11 subunit is a nuclease with both $5^{\prime}$ flap endonuclease activity and $3^{\prime} \rightarrow 5^{\prime}$ exonuclease activity and catalyses resection through cleaving the 5 ' strand, internal to the DSB, which is then resected using its exonuclease function to generate the short 3 ' single-strand (ss) DNA overhangs ${ }^{40}$. These overhangs are further resected by Exonuclease 1 (EXO1), forming long tracts of 3 ssDNA on either side of the DSB ${ }^{39}$. NBS1, the eukaryote specific component, is responsible for binding multiple phosphorylated proteins and recruiting MRE11 and RAD50 to DSB sites ${ }^{41}$ through its interaction with MRE11, CtIP, which is also required for initiating resection, and the ATM kinase ${ }^{42}$. End recognition and DSB processing by MRN is an ATP dependent process: here, ATP binding to RAD50 acts to switch the complex from an open to a closed conformation ${ }^{43}$, which facilitates DSB recognition, tethering and ATM activation ${ }^{43}$. In yeast the MRX complex also acts in telomere maintenance by binding the end of short telomeres and recruiting TEL1, which then recruits telomerase to extend the telomere ${ }^{44}$. Conversely, in mammalian cells, MRN regulates an ATM dependent response at dysfunctional telomeres ${ }^{45}$.

\section{RAD50 (Tb.927.11.8210), MRE11 (Tb927.2.4390) ${ }^{46,47}$ and NBS1 (Tb 927.8.5710) homologues are} present in the trypanosome genome and previous studies have shown that MRE11 is required for HR but its inactivation did not lead to telomere shortening or changes in VSG switching ${ }^{46,47}$, despite the dominance of $H R$ in repair in trypanosomes and requirement for the reaction in antigenic variation. What roles these proteins play in the trypanosome DDR is largely unexplored. In addition, though we know that DSBs accumulate at the subtelomeres ${ }^{26,27}$, it is unclear how they are sensed or how they 
contribute to antigenic variation. Given the central, early role of the MRN complex in DSB recognition and in telomere maintenance we set out to characterise its role in HR and VSG switching in trypanosomes. We found that RAD50, like MRE11, is required for efficient $H R$, and in its absence MMEJ dominated as the major form of repair. RAD50 also plays a perhaps surprising role in VSG switching, where it restricts HR substrate selection in the VSG repertoire and so may act to preserve the VSG archive during long-term infections.

\section{MATERIALS AND METHODS}

Trypanosoma brucei growth and manipulation. Lister 427, MITat1.2 (clone 221a), bloodstream stage cells were cultured in $\mathrm{HMI}-11$ medium ${ }^{79}$ at $37.4{ }^{\circ} \mathrm{C}$ with $5 \% \mathrm{CO}_{2}$. Cell density was determined using a haemocytometer. For transformation, $2.5 \times 10^{7}$ cells were spun for 10 minutes at $1000 \mathrm{~g}$ at room temperature and the supernatant discarded. The cell pellet was resuspend in prewarmed cytomix solution ${ }^{80}$ with $10 \mu \mathrm{g}$ linearised DNA and place in a $0.2 \mathrm{~cm}$ gap cuvette, and nucleofected (Lonza) using the X-001 program. The transfected cells were placed into one $25 \mathrm{~cm}^{2}$ culture flask per transfection with $36 \mathrm{ml}$ warmed $\mathrm{HMl}-11$ medium only and place in an incubator to allow the cells to recover for approximately 6 hours. After 6 hours, the media distributed into 48-well plates with the appropriate drug selection. Strains expressing TetR and I-Scel with I-Scel recognition-sites at a chromosome-internal locus ${ }^{17}$ and an active VSG-ESs ${ }^{27}$ have been described previously. G418, and blasticidin were selected at $10 \mu \mathrm{g} \cdot \mathrm{ml}^{-1}$ and $2 \mu \mathrm{g} \cdot \mathrm{ml}^{-1}$ respectively. Puromycin, phleomycin, G418, hygromycin and blasticidin and tetracycline were maintained at $1 \mu \mathrm{g} \cdot \mathrm{ml}^{-1}$. Clonogenic assay were plated out at either 32 cells per plate under both inducing and non-inducting conditions for ${ }^{1} \mathrm{HR}$ and VSG up strains and 480 cells per plate for VSGup strains under inducing conditions. Plates were counted 5-6 days later and subclones selected for further analysis.

To generate the RAD50 nulls in the ${ }^{1} \mathrm{HR}$ strain we employed multi-step transfection strategy ${ }^{81}$ that recycled a Neomycin phosphotransferase gene (NEO) in order to rescue one marker (here Blasticidin - BLA). Briefly, an I-Scel recognition sites was inserted into the pRAD50-BLA knock-out cassette between the 5'UTR and BLA ORF (Figure 1C) in the 2 T1 cell line ${ }^{82}$ with a tetracycline inducible Sce ORF. Induction of Sce induces a break in the BLA cassette and subsequent repair, using homology in the NEO modified allele, replaces BLA with NEO.

Clonogenic assays were plated out at either 32 cells per plate under both inducing and noninducing conditions for ${ }^{1} \mathrm{HR}$ and VSG up strains and 480 cells per plate for VSG ${ }^{\text {up }}$ strains under inducing conditions. Plates were counted 5-6 days later and subclones selected for further analysis.

Plasmid construction. For native $C$-terminal epitope tagging of Tb927.5.1700 / RPA2 a 765-bp fragment was amplified using primers RPA28F:GATCAAGCTTATGGAAGGAAGTGGAAGTAA; and RPA28R:GATCTCTAGAAATGCCAAACTTACAATCATG and cloned in PNAT ${ }^{\times T A G} 83$ using the HindIII and Xbal sites (underlined). The construct was linearized with Xhol prior to transfection. MRE11F5 (GATCgcggccgcATGGCCGAGAGGGCATC), MRE11R5 (GATCtctagaCAACGAAGATGTATGCCC), MRE11F3 (GATCgggeccCGATGGATAGTGGTAAT) and 
MRE11R3 (GATCggtaccCTAATAGTTATCTGGCA) were used to clone in target regions to generate PMRE11KOBLA and pMRE11KONEO. For transfection, $20 \mu \mathrm{g}$ pMRE11KO Blasticidin (BLA) and

160 Neomycin (NEO) plasmids were sequentially digested with Acc65I and Notl and cleaned by phenol-

161 chloroform extraction and ethanol precipitation after each digestion. Strains were validated using

162 MRE11F5 and MRE11R3 in a PCR assay. Heterozygous (+/-) and homozygous (-/-) knockout mutants

163 of RAD50 were generated by deleting most of replacing most of the gene's open reading frame with

164 either BLA and NEO. The strategy used is as described in ${ }^{84}$; briefly, two modified versions of the

165 plasmid pmt123 were used to allow PCR-amplified 5' and 3' flanking untranslated regions of RAD50 to

166 be inserted around BLA and NEO cassettes (where the antibiotic resistance genes' ORF were flanked

167 by tubulin and actin intergenic regions). The selective drug markers, flanked by RAD50 5' and 3'

168 untranslated regions, were then excised using Notl and transfected into $T$. brucei, and clones selected

169 using $10 \mu \mathrm{g} \cdot \mathrm{ml}^{-1}$ blasticidin or $5 \mu \mathrm{g} \cdot \mathrm{mL}^{-1} \mathrm{G} 418$.

Immunofluorescence microscopy. Immunofluorescence analysis was carried out using standard

172 protocols as described previously ${ }^{85}$. Mouse $\alpha-M y c$ was used at $1: 400$ and rabbit $\alpha-\gamma \mathrm{H} 2 \mathrm{~A}^{55}$ was used

173 at 1:250. Fluorescein-conjugated goat $\alpha$-rabbit and goat $\alpha$-mouse secondary antibodies (Pierce) were

174 used at 1:2000. Samples were mounted in Vectashield (Vector Laboratories) containing 4, 6-

175 diamidino-2-phenylindole (DAPI). In T. brucei, DAPI-stained nuclear and mitochondrial DNA can be

176 used as cytological markers for cell cycle stage ${ }^{86}$; one nucleus and one kinetoplast $(1 \mathrm{~N}: 1 \mathrm{~K})$ indicate

$177 \mathrm{G}_{1}$, one nucleus and an elongated kinetoplast ( $\left.1 \mathrm{~N}: \mathrm{eK}\right)$ indicate $S$ phase, one nucleus and two

178 kinetoplasts ( $1 \mathrm{~N}: 2 \mathrm{~K}$ ) indicate $\mathrm{G}_{2} / \mathrm{M}$ and two nuclei and two kinetoplasts ( $2 \mathrm{~N}: 2 \mathrm{~K}$ ) indicate post-mitosis.

179 Images were captured using a ZEISS Imager 72 epifluorescence microscope with an Axiocam 506

180 mono camera and images were processed and in ImageJ.

182 DNA analysis. Slot blots for detection of ssDNA were carried as described previously ${ }^{17}$. Image J was

183 used to generate linear density plots. The VSG probe was a 750 bp fragment VSG2 fragment from a

184 Pst1 digest of pNEG. The RFP probe was a 687-bp Hindll//Notl fragment encompassing the full ORF.

185 Loading control was a 226 - bp product from Tb427.01.570 (Dot1bKOF:

186 TGGTCGGAAGTTGGATGTGA Dot1bKOR: CTTCCATGCATAACACGCGA).

188 PCR analysis of RAD50 nulls to confirms knock-out were done using standard PCR conditions with

189 the following primers; a 402 bp product for RAD50 using RAD50KOF

190 (CGTGAGAAACAGGAACAGCA) and RAD50KOR (AACACGTTTTTCCAACTCGG); a 399 bp product

191 for Blasticidin ORF using BlaF (GATCGAATTCATGGCCAAGCCTTTGTCT) and BlaR

192 (GATCCCATGGTTAGCCCTCCCACACATAA); and a 795 bp product for Neomycin

193 Phosphotransferase ORF using NPTF (ATGATTGAACAAGATGGATTG) and NPTR

194 (TCAGAAGAACTCGTCAAGAA). Analysis of subclones was previously described ${ }^{21,27,35}$ and used the 195 following primers VSG221F (CTTCCAATCAGGAGGC), VSG221R (CGGCGACAACTGCAG), RFP

196 (ATGGTGCGCTCCTCCAAGAAC), PAC (TCAGGCACCGGGCTTGC), ESAG1F 

(GGGGTGAATGTTGGCTGTG), 2110Y (GGGATTCCCAGACCAATGA)

VSG sequencing analysis. For the RT-PCR, the reaction mix were as following; $1 \mu \mathrm{g}$ of $\mathrm{CDNA}, 1 \mathrm{x}$ PCR buffer, $0.2 \mathrm{mM}$ dNTPs, $1 \mu \mathrm{l}$ each of SL (ACAGTTTCTGTACTATATTG) and SP6-14mer (GATTTAGGTGACACTATAGTGTTAAAATATATC) primers, $\mathrm{H} 2 \mathrm{O}$ to $50 \mu \mathrm{l}$ and $0.5 \mu \mathrm{l}$ Phusion polymerase (New England Biolabs). For the PCR conditions. Five cycles were carried out at $94{ }^{\circ} \mathrm{C}$ for $30 \mathrm{~s}, 50^{\circ} \mathrm{C}$ for $30 \mathrm{~s}$ and $72{ }^{\circ} \mathrm{C}$ for $2 \mathrm{~min}$; followed by 18 cycles at $94^{\circ} \mathrm{C}$ for $30 \mathrm{~s}, 55^{\circ} \mathrm{C}$ for $30 \mathrm{~s}$ and $72{ }^{\circ} \mathrm{C}$ for 2 min. DNA concentration was measured using a Nanodrop. Libraries were prepared from VSG PCR products and sequenced on a BGI-Seq (BGI500) with a 150 bp paired-end read length with BGI Genomics Hong Kong.

Replicate libraries for WT uninduced, WT induced, VSGUP uninduced and VSGUP induced, VSGUP rad50 uninduced and VSGUP rad50 induced were sequenced on the BGlseq500 platform producing $8.03,9.01,7.60,7.22,6.66,7.07,6.90,6.98$ million reads per library, respectively. Reads were aligned to the $T$. brucei Lister 427 genome ${ }^{15}$ with the cohort of minichromosomal VSGs added from the Lister 427 VSGnome ${ }^{13}$ using bowtie ${ }^{87}$ with the parameters --very-sensitive and BAM files created with samtools ${ }^{88}$, aligning (WT uninduced) 97.76, 98.42, (WT induced) 97.69, (VSG ${ }^{\text {UP }}$ rad50 uninduced) $97.57,98.60$, (VSG UP rad50 induced) $97.63,97.80$ percent of reads successfully. Reads counts per transcript were obtained using featureCounts ${ }^{89}$. Differential expression analysis was performed using Edge ${ }^{90}$ on all genes, followed by filtering for VSG genes (1848 VSG sequences in total). An R script (https://github.com/LGloverTMB/DNA-repair-mutant-VSG-seq ) was used to perform differential expression analysis, and generate Volcano and genome scale plots. BLAST analysis was performed locally using a database containing significantly up-regulated VSG genes from both conditions, including $2 \mathrm{~kb}$ of sequence upstream and downstream of the start and stop codons, respectively (except where sequences in the contigs 5' or 3' to the CDS were shorter than $2 \mathrm{~kb}$ excluding). For this analysis, minichromosomal VSG genes were excluded as the VSGnome does not contain any sequence beyond the CDS. The resulting database of 131 VSGs was queried using the VSG2 sequence including $2 \mathrm{~kb}$ of sequence upstream of the CDS and all sequence between the stop codon and end of contig (1,272 nt). The BLASTn algorithm was used query the database using default parameters except allowing up-to 20 hits per subject sequence, and outputting up to 2,000 alignments.

227 Alignments were filtered to remove overlapping hits from the same subject sequence using Microsoft

228 Excel, retaining the one with the higher alignment score. Non-overlapping alignments were plotted 229 using a custom R script (https://github.com/LGloverTMB/DNA-repair-mutant-VSG-seq). Lengths of 230 average alignments were calculated for cohorts of VSGs up-regulated in both VSGup and VSG up rad50 231 or VSGup rad50 only. 


\section{RESULTS}

RAD50 is required for normal cell growth and DSB repair.

RAD50, the largest component of the MRN complex, belongs to the structural maintenance of chromosomes (SMC) family of proteins ${ }^{48}$ and has not been examined in T. brucei, though the gene has been reported to be essential in Leishmania infantum ${ }^{49}$. The domain architecture of RAD50 is approximately palindromic (Figure $1 \mathrm{~A}$ ) and characterized by the presence of ATP-binding cassette (ABC)-ATPase domains at the $\mathrm{N}$ - and $\mathrm{C}$ - termini, each followed by an MRE11 binding site (MBS), and then by anti-parallel coiled-coil regions, which form linker structures that enable the MRN complex to act as a tethering scaffold to hold broken chromosomes together for repair ${ }^{50}$. Between the antiparallel coiled-coils, a central Zn hook, a CxxC motif, facilitates $\mathrm{Zn}^{2+}$ dependent RAD50-RAD50 subunit interactions and is presumed to be important for tethering ${ }^{51}$. A conformational change is invoked through binding of RAD50 to two ATP molecules, which then allows for binding to DNA ${ }^{43}$. Primary sequence comparison suggested all RAD50 domains are recognisably conserved in the putative $T$. brucei RAD50 homologue (Tb.927.11.8210; Supplementary Figure 1). Within the ATPase domains, the $A B C$ nucleotide binding domain is defined by the conserved presence of Walker $A, Q$-loop, Signature, Walker B, D-loop, and H-loop motifs required to form the active ATPase site ${ }^{52}$. Furthermore, structure prediction using Phyre ${ }^{23}$ modelled 503 residues ( $37 \%$ of the sequence) of the T. brucei protein, revealing a SMC head domain and antiparallel coiled coil regions (Figure 1A).

To test the function of RAD50 in DSB repair, we used a previously validated T. brucei cell line, referred to as ${ }^{1} \mathrm{HR}$ (Figure 1B), where a single I-Scel meganuclease DSB can be induced in an RFP$P A C$ (red fluorescent protein - puromycin $\mathrm{N}$-acetyltransferase) fusion cassette in the core region on chromosome $11^{17}$ (Figure 1B). We generated rad50 null mutants (referred to as ${ }^{1} \mathrm{HR}$ rad50) in these cells by sequentially replacing the two gene alleles with neomycin phosphotransferase (NEO) and blasticidin $(B L A)$ resistance cassettes: PCR analysis of double antibiotic resistant clones confirmed RAD50 loss and replacement (Supplementary Figure $2 A$ and $B$ ) and demonstrates RAD50 is not essential in $T$. brucei. To determine the role RAD50 plays in DNA repair, we set up clonogenic assays. Cells were distributed across 96 -wells plates under both I-Scel non-inducing and inducing conditions, and wells with live cells scored after $5-7$ days. This revealed a significant growth defect in the ${ }^{1} \mathrm{HR}$ rad50 null cells in unperturbed cells (Figure 1C Left panel and Table 1): $95 \%$ of the WT ${ }^{1} \mathrm{HR}$ cells survived compared with $\sim 35 \%$ of the ${ }^{1} \mathrm{HR}$ rad50 cells, revealing a 2.6 - fold decrease in cell survival. This growth impairment is likely due to the inability to repair spontaneous DSBs. Induction of the I-Scel meganuclease results in $\sim 95 \%$ cutting and repair mainly by homologous recombination ${ }^{17}$. Consistent with previous findings, in the WT ${ }^{1} \mathrm{HR}$ strain $\sim 48 \%$ of cells are able to repair the DSB and survive (Figure 1C Left panel and ${ }^{17}$ and Table 1), whereas in the ${ }^{1} \mathrm{HR}$ rad50 cells, a severe growth defect was seen following a DSB, with less than $3 \%$ survival (a 16 - fold reduction), suggesting a significant defect in DSB repair (Figure 1C Left panel and Table 1). This was recapitulated when assessing the normalised survival efficiency (compared to uninduced survival) following an I-Scel break (Figure 1C 
right panel and Table 1), indicating that a DSB is more lethal in the null mutant cells. In parallel we also tested the function of MRE11, as it forms a complex with RAD50, by generating null mutants through sequentially replacing the two gene alleles with NEO and BLA resistance cassettes; PCR analysis confirmed MRE11 loss and replacement (Supplementary Figure 2C). The mre11 nulls (referred to as ${ }^{1} \mathrm{HRmre11}$ ) also showed a growth defect cells in the unperturbed cells, with only $47 \%$ of cells surviving cloning. Induction of the I-Scel meganuclease resulted in a severe growth defect, with less than $2 \%$ survival, suggesting a significant defect in DSB repair (Supplementary Figure 4A and Table 1) whose magnitude was very similar to ${ }^{1} \mathrm{HRrad} 50$ cells, which is expected given they have been shown to act in complex in other systems ${ }^{37,38}$.

A
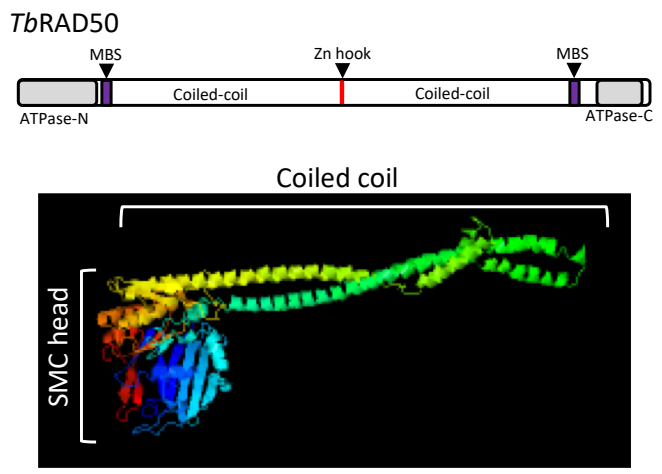

B

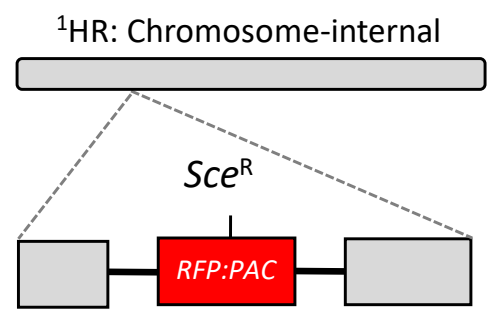

C

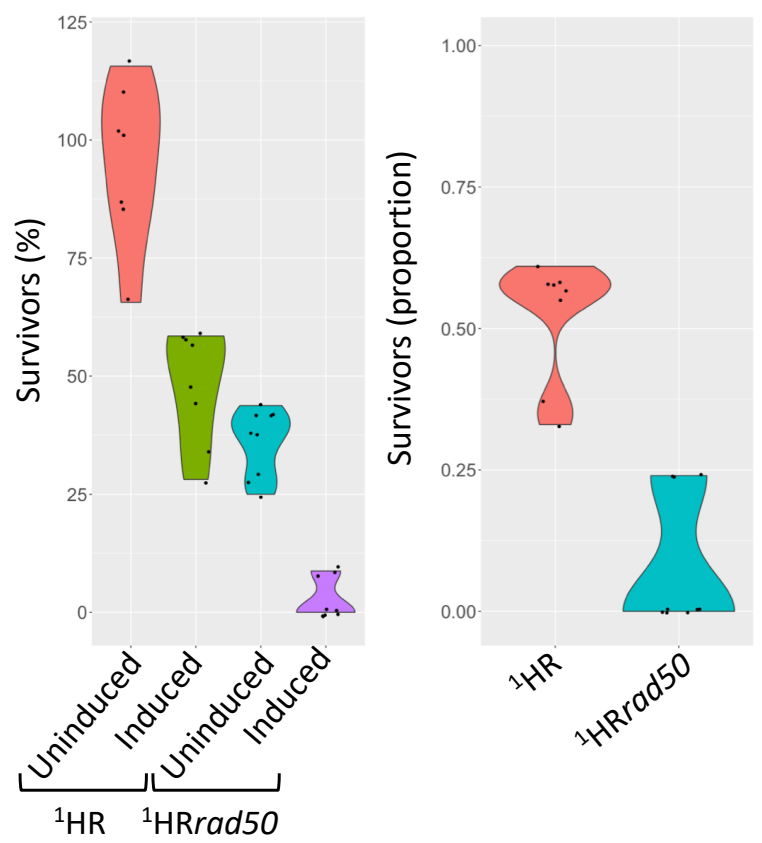

282

Figure 1: RAD50 is essential for DSB response and repair at a chromosome-internal locus. (A) Upper panel: Schematic of TbRAD50 with protein domains. Amino acid position of conserved domains are: ATPase - N, 4 - 170; MRE11 binding site (MBS), 182 - 205; Zn hook, 690 - 693; MRE11 binding site, 1158 - 1181; ATPase - C, 1243 - 1333. Lower panel: The structure of the T. brucei RAD50 was modelled using Phyre2 showing the SMC head domain with a coiled coil. (B) Schematic of the chromosome-internal DSB cell line with the I-Scel recognition site, Sce ${ }^{R}$, highlighted. (C) A clonogenic assay reveals survivors following a DSB at a chromosome-internal locus in the parental and ${ }^{1} \mathrm{HRrad50}$ cell lines. Cells were plated out into media with or without tetracyline. The proportion of survivors was calculated by dividing the number of induced survivors by uninduced. R:P, red fluorescent protein: puromycin fusion gene. ${ }^{1} \mathrm{HR}$ technical replicates; $\mathrm{n}=2$, and with ${ }^{1} \mathrm{HR}$ rad50 biological replicates for the strains; $n=2$. 

trypanosomes recognize a DSB lesion and initiate a signalling cascade resulting in DNA repair ${ }^{54}$. In

298 T. brucei, the DDR after an I-Scel induced DSB has been characterized thus far to include an increase 299 of cells in $\mathrm{G}_{2} / \mathrm{M}^{17}$, phosphorylation of histone $\mathrm{H}_{2} \mathrm{~A}^{55}$, break resection and accumulation of RAD51 foci 300 at the site of the DSB ${ }^{17}$. The cell cycle distribution of WT ${ }^{1} \mathrm{HR}$ and ${ }^{1} \mathrm{HR}$ rad50 cells was assessed 301 following induction of a DSB. In WT ${ }^{1} \mathrm{HR}$ cells $\sim 28 \%$ were in $\mathrm{G}_{2} 12$ hours after I-Scel induction and 302 this returned to background levels ( $15 \%$ of the population) by 24 hours. In contrast, no increase in $\mathrm{G}_{2}$ 303 cells was seen after DSB induction in the ${ }^{1} \mathrm{HR}$ rad50 cells (Figure $2 \mathrm{~A}$ ), suggesting RAD50 is required 304 for eliciting the $G_{2} / M$ checkpoint. In mammals the MRN complex recruits the ATM kinase to a DSB, 305 where it phosphorylates $\mathrm{H}_{2} \mathrm{AX}^{37}$.

306

A

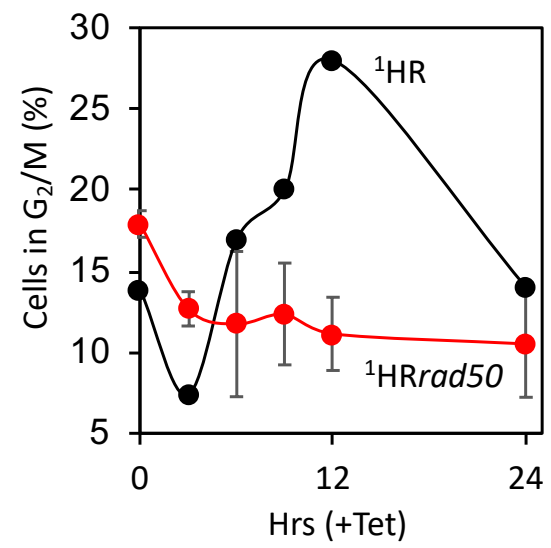

B

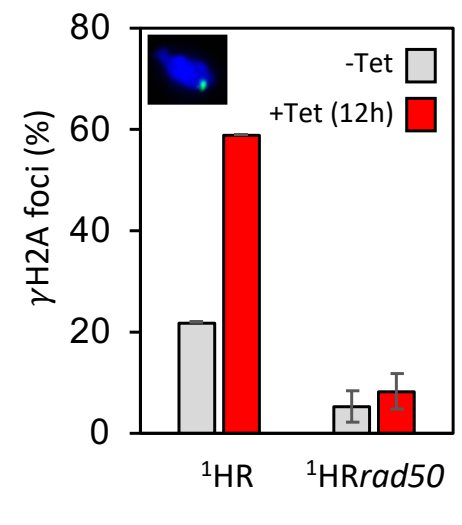

C

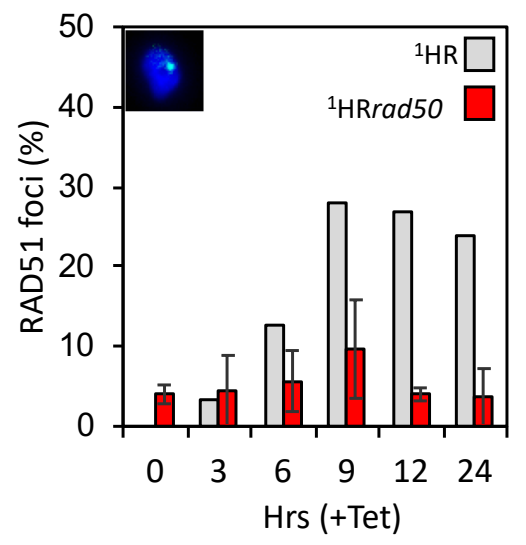

307

308

309

310

311

312

313

314

315

316

317

Figure 2: DNA damage response is compromised in ${ }^{1} \mathrm{HR}$ rad50 cells. (A) The number of cells in G2/M phase cells was counted by DAPI staining at several points following induction of an I-Scel break in.

$\mathrm{G} 2$ cells contain one nucleus and two kinetoplasts. (B) Immunofluorescence assay to monitoring $\gamma \mathrm{H} 2 \mathrm{~A}$ foci. The number of positive nuclei were counted in uninduced cells and 12 hours post DSB. Inset showing a nucleus with a $\gamma \mathrm{H} 2 \mathrm{~A}$ focus. $n=200$ for each time point in the ${ }^{1} \mathrm{HR}$ cell line and $n=400$ for the ${ }^{1} \mathrm{HR}$ rad50 strain. Error bars, SD, for ${ }^{1} \mathrm{HR}$ rad50 biological replicates for the strains; $\mathrm{n}=2$. (C) Immunofluorescence assay to monitoring RAD51 foci. The number of positive nuclei were counted in uninduced cells and 12 hours post DSB. Inset showing a nucleus, with a single RAD51 focus. $n=200$ for each time point in the ${ }^{1} \mathrm{HR}$ cell line and $n=400$ for the ${ }^{1} \mathrm{HR}$ rad50 strain. Error bars, SD, for ${ }^{1} \mathrm{HR}$ rad50 biological replicates for the strains; $\mathrm{n}=2$.

Using an antibody specific to the Thr130 phosphorylated form of $T$. brucei $\mathrm{H} 2 \mathrm{~A}, \gamma \mathrm{H} 2 \mathrm{~A}{ }^{55}$, we saw the expected background staining of $\sim 15-20 \%$ of nuclei with foci in unperturbed WT ${ }^{1} \mathrm{HR}$ cells, which increased to $\sim 60 \%$ at 12 hours post I-Scel induction (Figure $2 \mathrm{~B}$ ). In the ${ }^{1} \mathrm{HR}$ rad50 cells, the background level of $\gamma \mathrm{H} 2 \mathrm{~A}$ foci was reduced to $5 \%$, and the DSB-induced increase was drastically impaired (Figure $2 \mathrm{~B}$ ), with only $8 \%$ of cells containing $\gamma \mathrm{H} 2 \mathrm{~A}$ foci. Repair at this locus is predominately via RAD51-dependent homologous recombination ${ }^{17}$, and so we next assessed RAD51 foci assembly 
following DSB induction. In the WT ${ }^{1} \mathrm{HR}$ strain, the number of detectable foci increased from 0 to $27 \%$ within $9-12$ hours after I-Scel induction. In contrast, in ${ }^{1} \mathrm{HR}$ rad50 cells the background level of

327 RAD51 foci, before I-Scel induction, was higher at $4 \%$, and only increased to $10 \%$ ( 3 fold reduced)

328 in response to a DSB (Figure 2C). Like the ${ }^{1} \mathrm{HRrad50}$ cells, we detected fewer $\gamma \mathrm{H} 2 \mathrm{~A}$ foci in ${ }^{1} \mathrm{HRmre} 11$ cells following a DSB (no foci detected, Supplementary Figure 4B) and a significant reduction in the number of RAD51 foci (14\% compared with $36 \%$ in WT, Supplementary Figure 4B) and a loss of the $\mathrm{G}_{2} / \mathrm{M}$ checkpoint (8.5\% compared with $28 \%$ in WT, Supplementary Figure $4 \mathrm{~B}$ ). These results reveal an important role for RAD50 and MRE11 in the DDR to a DSB in trypanosomes at a single copy locus and suggest wider roles in tackling spontaneous DNA damage.

RAD50 restricts resection during allelic recombination.
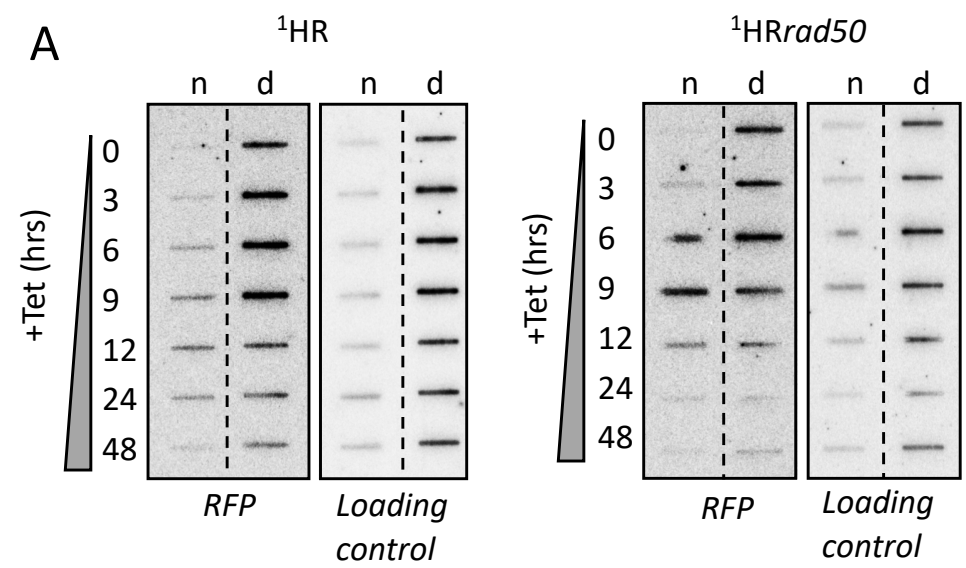

B

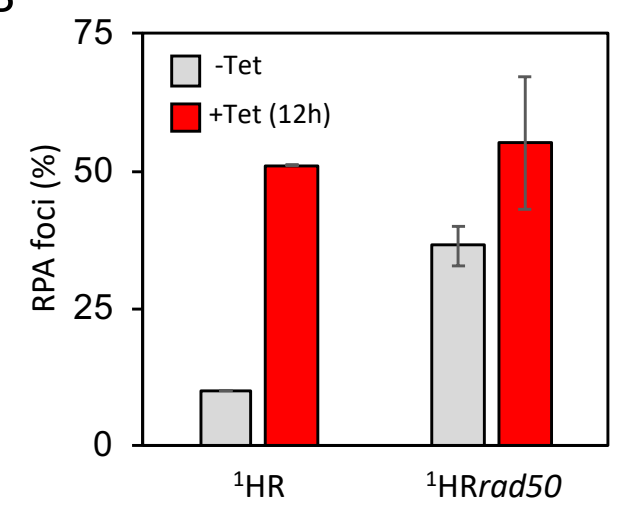

335

336

337

338

339

340

341

342

343

344
C

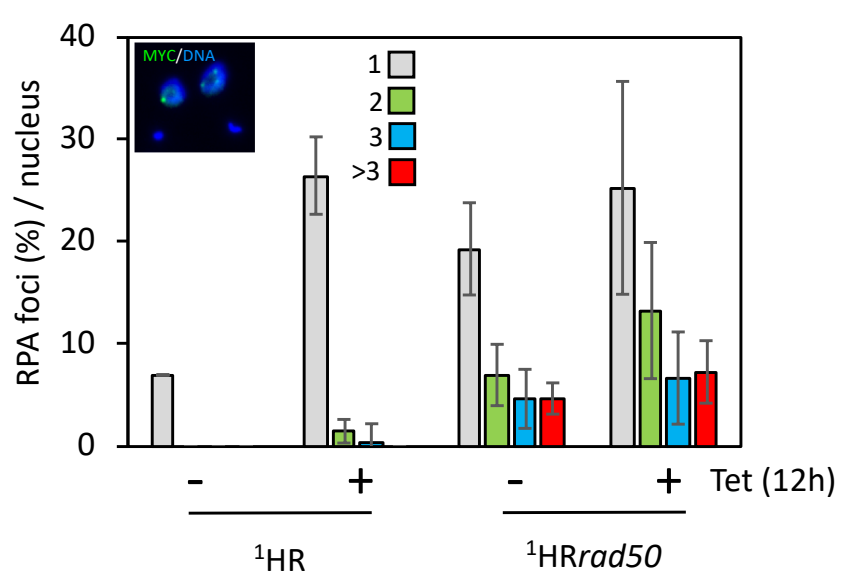

Figure 3: RAD50 directs resection at chromosome-internal locus. (A) Accumulation of SsDNA was monitored using slot-blots. Genomic DNA was extracted as indicated following I-Scel induction. Ninety percent of the sample was 'native' ( $\mathrm{n}$; ssDNA) and ten percent was denatured (d). Probe RFP and the loading control are described in the materials and methods. (B) Immunofluorescence assay to monitoring RPA foci. The numbers of positive nuclei were counted in uninduced cells and 12 hours post DSB. $n=200$ for each time point in the ${ }^{1} \mathrm{HR}$ cell line and $n=400$ for the ${ }^{1} \mathrm{HR}$ rad50 strain. Error bars, SD, for ${ }^{1} \mathrm{HRrad50}$ biological replicates for the strains; $n=2$. (C) Immunofluorescence assay to monitor the number of RPA foci per nucleus. The number of RPA foci was counted in uninduced cells and 12 hours post DSB. $n=200$ nuclei for each time point in the ${ }^{1} \mathrm{HR}$ cell line and $n=400$ nuclei for 
the ${ }^{1} \mathrm{HR}$ rad50 cells. Inset showing representative nuclei, with RPA foci. Error bars, SD, for ${ }^{1} \mathrm{HRrad50}$ biological replicates for the strains; $\mathrm{n}=2$.

An early step in the DSB repair cycle is the formation of extensive 3' ssDNA overhangs, initiated by MRE11 3' - 5' nuclease activity, which are a substrate for RAD51 nucleoprotein filament formation and act as a template for homology-directed repair ${ }^{37}$. In light of the reduced accumulation of RAD51 foci after DSB induction in the absence of RAD50, we sought to determine whether the formation of ssDNA at the I-Scel target locus was compromised, using slot blots. In the WT ${ }^{1} \mathrm{HR}$ cells, ssDNA accumulated up to $12 \mathrm{~h}$ after I-Scel induction and declined thereafter (Figure $3 \mathrm{~A}$ ), mirroring the phosphorylation of H2A and accumulation of RAD51 (Figure $2 B$ and $C)^{17}$. Processing of the DSB in the ${ }^{1} \mathrm{H}$ Rrad50 cells appeared to be accelerated, with ssDNA signal peaking at 9 hours and declining thereafter (Figure 3A and Supplementary Figure 3). We conclude that DNA resection is not lost in the ${ }^{1} \mathrm{HRrad} 50$ cells but the timing is affected, though we cannot say if the extent of resection is changed. Prior to RAD51 loading on to ssDNA, the trimeric RPA (replication protein A) complex binds the ssDNA and is subsequently displaced by RAD51 ${ }^{56}$. Rescue of the $B L A$ selectable marker in this strain (Supplementary Figure 2) allowed tagging of RPA2 with the myc epitope and subsequent localization. In WT ${ }^{1} \mathrm{HR}$ cells the number of nuclei with RPA foci increased 5 -fold (from $10 \%$ to $50 \%$ ) following an IScel break (Figure $3 \mathrm{~B}$ ). The ${ }^{1} \mathrm{HR}$ rad50 cells showed a pronounced increase in RPA foci prior to induction of a DSB, and only a marginal increase at 12 hours post DSB ( $30 \%-55 \%$; Figure $3 B)$. In the WT ${ }^{1} \mathrm{HR}$ cells, a single RPA focus is most commonly seen in response to an I-Scel break ${ }^{22}$. However, we observed multiple RPA foci in both induced and uninduced ${ }^{1} \mathrm{HR}$ rad50 null cells (Figure $3 \mathrm{C})$. We therefore tentatively conclude that most RPA signal in the ${ }^{1} \mathrm{HRrad} 50 \mathrm{cells}^{22}$ represents persistent, widespread damage, meaning it is unclear if loss of RAD50 alters the accumulation of RPA at the I-Scel induced DSB in chromosome 1.

RAD50 is crucial for homologous recombination in T. brucei

To explore how trypanosomes repair a DSB in the ${ }^{1} \mathrm{HR}$ rad50 nulls, DSB-survivors from the clonogenic assay were scored for repair by homologous recombination or MMEJ by a PCR assay (Figure 4A) using sets of primers that flanked the RFP-PAC cassette. In the surviving subclones, sensitivity to puromycin is indicative of cleavage by I-Scel ${ }^{17}$. All twelve of the surviving subclones were sensitive to puromycin, indicating cleavage by $\mathrm{I}-\mathrm{Scel}{ }^{17}$ and disruption of the RFP:PAC cassette (data not shown). Eleven of these clones showed repair by MMEJ, as seen by the reduction in the size of the PCR product as compared to the controls (Figure 4B, RFP and PAC primer pair ${ }^{17}$ ), or loss of the entire cassette (Figure 4C, 2110X and 2110Y primer pair ${ }^{57}$ ). Sequencing revealed repair by MMEJ using the $X \mathrm{~cm} 1$ sites that flanked the RFP-PAC cassette in clones 12, 15, 16 and 17 (Figure 4C lower panel). Sequencing of the $2110 X-Y$ product in clone 8 revealed repair using the homologous template, suggesting homologous recombination can still occur, although is significantly impaired. In the mre11 nulls, 14 out of 15 clones repaired by MMEJ (Supplementary Figure 4C, clone 10, 17 and 19 show a PCR product of reduced size and for the remaining clones the 2110XY PCR product was sequenced revealing 11 clones had repaired by MMEJ). These data show a significant shift in the pathway used 
to repair a DSB in the ${ }^{1 \mathrm{~s}} \mathrm{HRrad} 50$ and ${ }^{1} \mathrm{HRmre} 11$ null cells at a chromosome-internal locus, with repair 386 by MMEJ dominating (Figure 4D), compared with the pronounced predominance of homologous 387 recombination in $\mathrm{WT}^{1} \mathrm{HR}$ cells ${ }^{17}$.

A

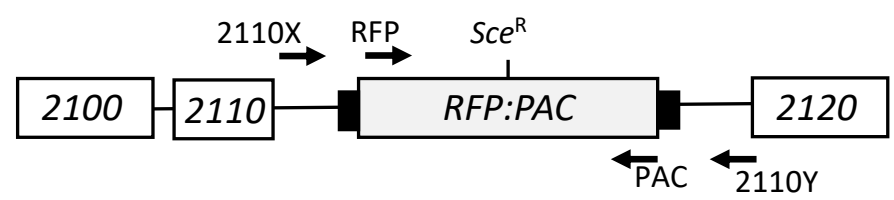

B

${ }^{1} \mathrm{HR}$ rad50 subclones

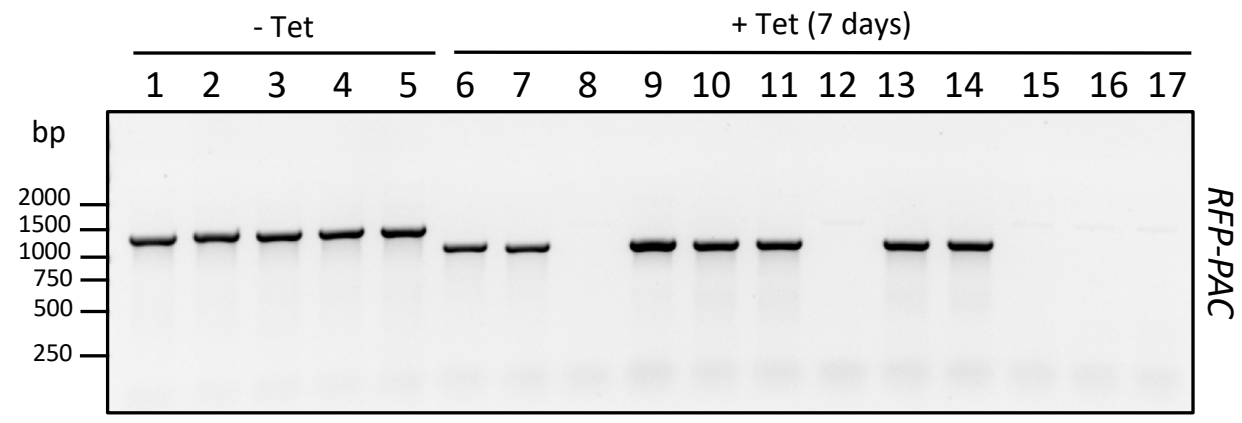

C

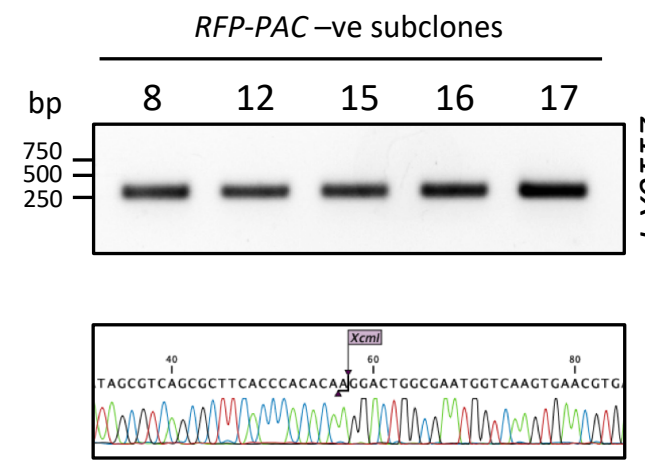

D

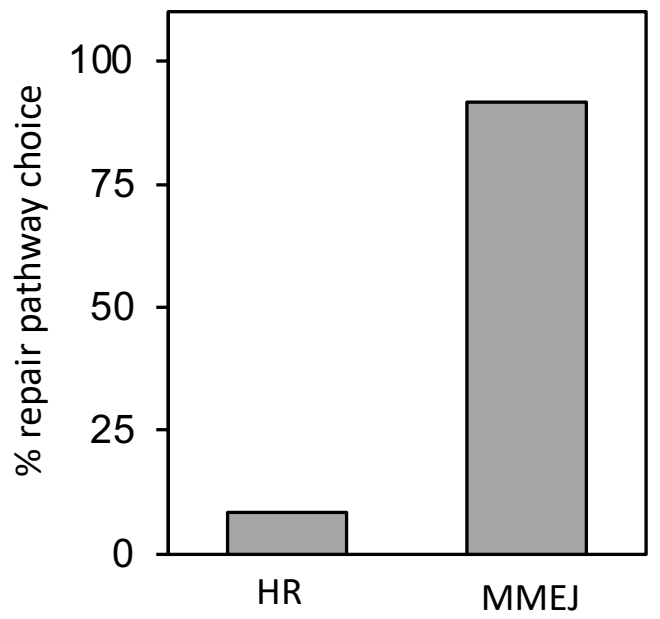

389 Figure 4: RAD50 is required for homologous recombination. PCR analysis of ${ }^{1} \mathrm{HR}$ repaired

390 subclones. (A) Schematic showing the 2110 locus and position of the Sce recognition site (Sce ${ }^{\mathrm{R}}$ ).

391 Position of primers indicated by arrows. Primer sequence detailed in materials and methods. (B). PCR 392 assay of repaired subclones showing RFP:PAC presence or absence. (C) Upper panel: PCR assay of 393 repaired subclones that were negative for RFP:PAC. Lower panel: Sanger Sequence trace showing 394 Xcml site. (D) Percentage of survivors for each repair pathway choice. $n=12$ clones. Arrows indicate 395 position of primers. White box, genes; Grey box, RFP - PAC fusion gene; black box, UTRs.

396 Loss of RAD50 increases survival following a DSB at the active VSG-ES. 
Trypanosomes rely on homologous recombination to facilitate antigenic variation. We therefore wanted to test the role of RAD50 in VSG switching. We generated RAD50 nulls in a cell line where the I-Scel recognition site is fused to a puromycin selectable marker and inserted immediately downstream of the major block of 70-bp repeats and upstream of VSG2 in Bloodstream form Expression site 1 (BES1) (on chromosome 6a), the active VSG-ES in this strain (Figure 5A). The resulting cell line is known as VSG ${ }^{27}$. Cell survival following a DSB at this position is contingent upon VSG switching, most commonly using the 70-bp repeats and replacing the active VSG via breakinduced replication ${ }^{26,27}$. We generated rad50 null VSG up strains (VSG ${ }^{\text {up }}$ rad50), by replacing the two gene alleles with NEO and BLA resistance cassettes: PCR analysis of double antibiotic resistant clones confirmed RAD50 loss and replacement (Supplementary Figure $2 \mathrm{~A}$ and $\mathrm{B}$ ). Using a clonogenic assay we found that, like in the ${ }^{1} \mathrm{HR}$ strain, there was a growth defect (1.6-fold reduction) in the VSGup rad50 nulls compared with VSGup WT cells in the absence of I-Scel induced damage (Figure 5B; left panel and Table 1). This effect again suggests an impaired ability to repair spontaneous damage within the mutant cell. However, quite differently to ${ }^{1} \mathrm{HR}$ cells, following induction of an I-Scel DSB we observed an increase in survival in the VSG ${ }^{\text {up }}$ rad50 nulls compared with induced VSG ${ }^{\text {up }}$ WT cells (Figure 5B; left panel and Table 1). These data indicate that RAD50 suppresses DSB repair at a VSG-ES, the opposite of its role at a chromosome-internal DSB. In contrast, survival is reduced in the VSG ${ }^{\text {up }}$ mre11 nulls relative to VSG up WT, with only $1.75 \%$ able to survive a DSB (Supplementary Figure $6 \mathrm{~A}$ and Table 1). We then assessed the DDR in the VSGup cell line. As in the ${ }^{1} \mathrm{HR}$ cells, following a DSB, the number of cells that accumulate in $\mathrm{G}_{2} / \mathrm{M}$ increases to $~ 30 \%$ in the VSG ${ }^{\text {up }} \mathrm{WT}$ cell line ${ }^{27}$, and this cell cycle checkpoint was lost in the VSG ${ }^{\text {up }}$ rad50 cells (Figure $5 \mathrm{C}$ ) and in the VSG ${ }^{\text {up }}$ mre 11 cells (Supplementary Figure 6B). In the VSG ${ }^{\text {up }}$ WT cell line, the number of $\gamma \mathrm{H} 2 \mathrm{~A}$ foci increased from $20 \%$ to $41 \%$ after I-Scel induction, as has been previously reported ${ }^{27}$. In the VSG ${ }^{\text {up }}$ rad50 nulls, $\gamma \mathrm{H} 2 \mathrm{~A}$ foci were only detected in $3 \%$ of uninduced cells, and this increased to $11 \%$ following a DSB (Figure 5D). A similar phenotype was seen in the VSG ${ }^{\mathrm{up}} m r e 11$ cells, with the number of $\gamma \mathrm{H} 2 \mathrm{~A}$ foci increasing from $1.3 \%$ to $7 \%$ (Supplementary Figure $6 \mathrm{~B}$ ). Thus, while it appears that loss of RAD50 or MRE11 diminishes the capacity of cells to phosphorylate H2A in response to a DSB at this locus, the increased survival in the VSG ${ }^{\text {up }}$ rad50 cells suggests that while it is required for an efficient DDR, in its absence the cells are more adept at repair.

Using a series of assays (Figure 6A and Supplementary figure 5) we next looked at DNA rearrangements in the VSG-ES to determine how the VSG ${ }^{\text {up }}$ rad50 cells repair an induced DSB. 25 subclones were selected from the clonogenic assay and tested for sensitivity to puromycin, asking about the frequency of loss of the puromycin gene from the VSG-ES. 24 out of 25 subclones were sensitive to $1 \mu \mathrm{g} \cdot \mathrm{ml}^{-1}$ puromycin, indicating that the majority of the population had been subject to a DSB and had deleted the resistance cassette. Immunofluorescence using antibodies against VSG2, the VSG expressed from the modified VSG-ES, showed that 23 out of 24 puromycin sensitive subclones had switched VSG (Figure 6B). This is comparable to what is seen in the VSG ${ }^{\text {up }}$ WT strain, suggesting loss of RAD50 does not affect the cell's ability to undergo VSG switching. The single

436 puromycin resistant clone was VSG2 positive, suggesting I-Scel did not cut (Supplementary Figure 5, 437 subclone 14). 
A

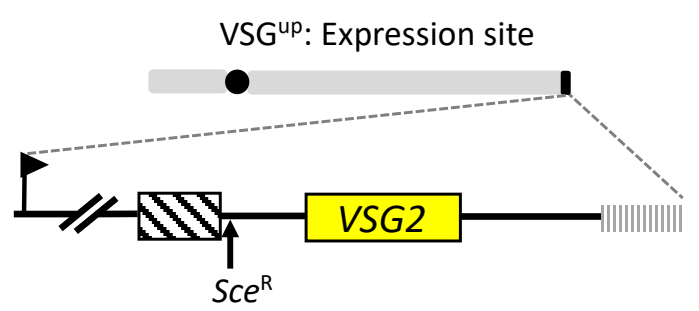

B

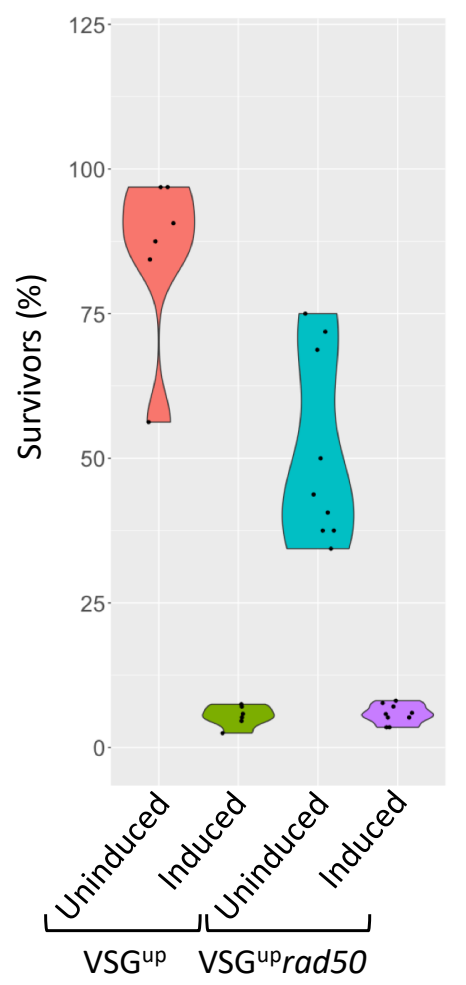

C

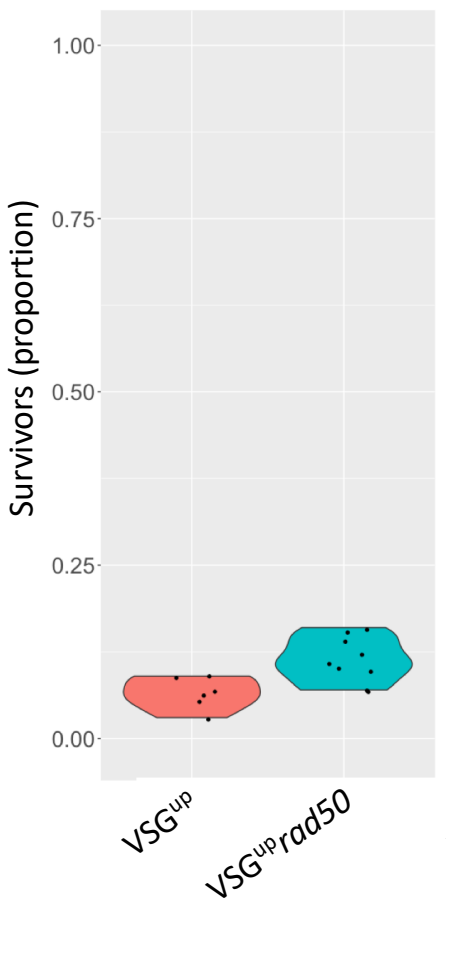

D
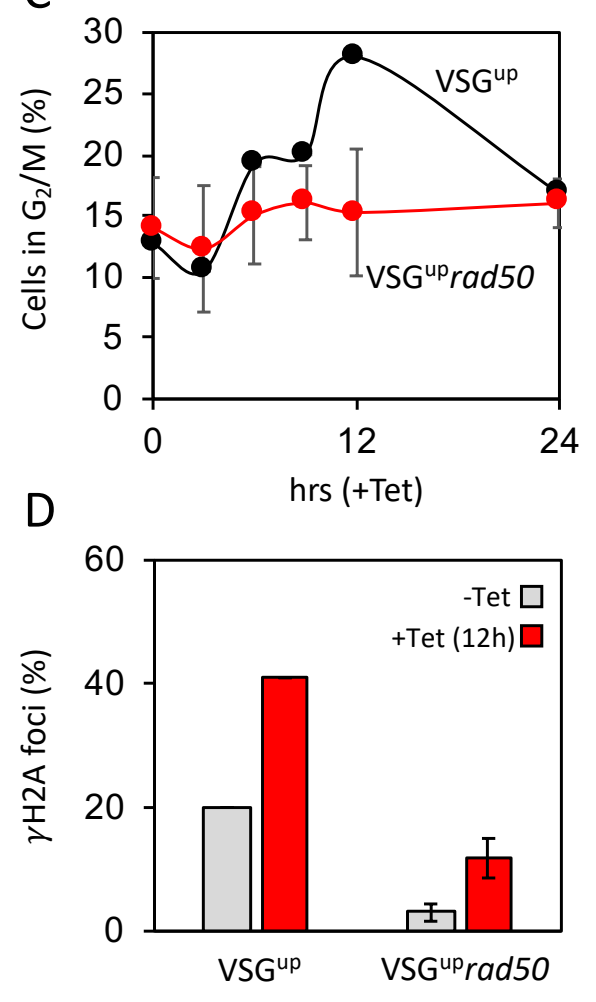

Figure 5: RAD50 suppresses repair at a subtelomeric locus. (A) A schematic of the active expression site DSB cell line with the I-Scel recognition site, $S c e^{R}$, highlighted. (B) A clonogenic assay reveals the survivors following a DSB at the active expression site in the parental and VSGUP rad50 cell lines. Cells were plated out into media with or without tetracyline and counted after seven days. Other details as in Figure 1. (C) The number of cells in G2/M phase cells was counted by DAPI staining at 0 hours and 12 hours following induction of an I-Scel break in. G2 cells contain one nucleus and two kinetoplasts. (D) Immunofluorescence assay to monitoring $\gamma \mathrm{H} 2 \mathrm{~A}$ foci. The nuclei with $\gamma \mathrm{H} 2 \mathrm{~A}$ foci were counted in uninduced cells and 12 hours post DSB. $n=200$ for each time point in the VSG up cell line and $n=400$ for the RAD50 null. Error bars, SD, for VSGup rad50 biological replicates for the strains; $\mathrm{n}=2$. Arrow, RNA Pol 1 promoter; box with diagonal lines, 70-bp repeats; vertical lines, telomere. We then looked at DNA rearrangements in the ES using primers specific to VSG2 and ESAG1.

452 ESAG1 is found upstream of a block of 70-bp repeats in the active VSG-ES and cells that retain $453 E S A G 1$ are presumed to have repaired by gene conversion using the 70-bp repeats. Both VSG2 and ESAG1 were retained in five out of five uninduced control subclones (Figure 6C and Supplementary 
Figure 3). Of the 24 puromycin sensitive subclones (i.e. cleaved by I-Scel), ESAG1 was retained in 23

456 and VSG2 was lost in 23 (Figure 6C and Supplementary Figure 5).

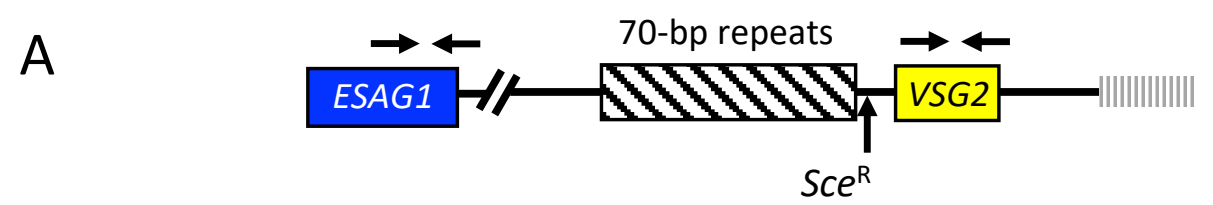

B

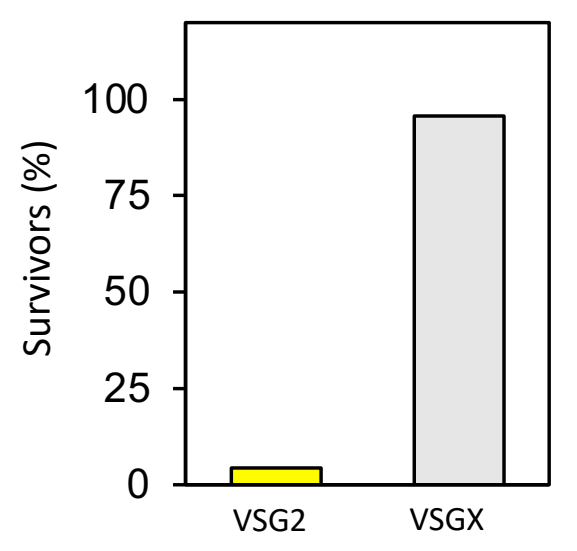

D

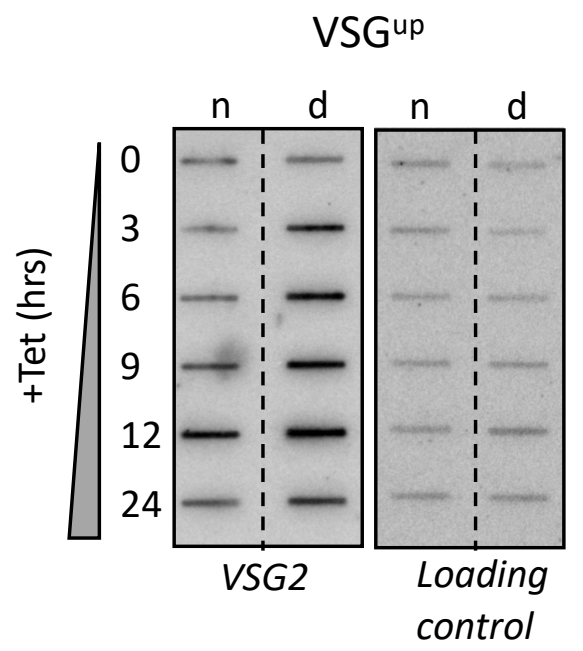

C

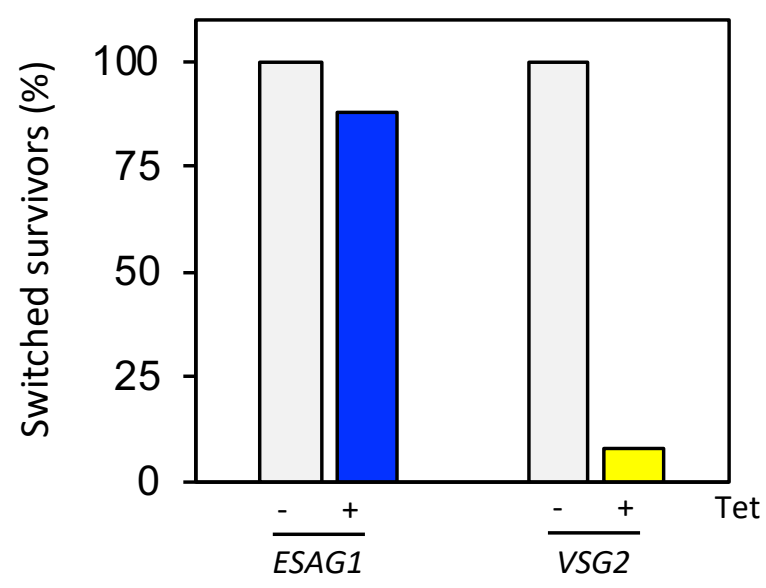

VSG ${ }^{\mathrm{up}}$ rad50

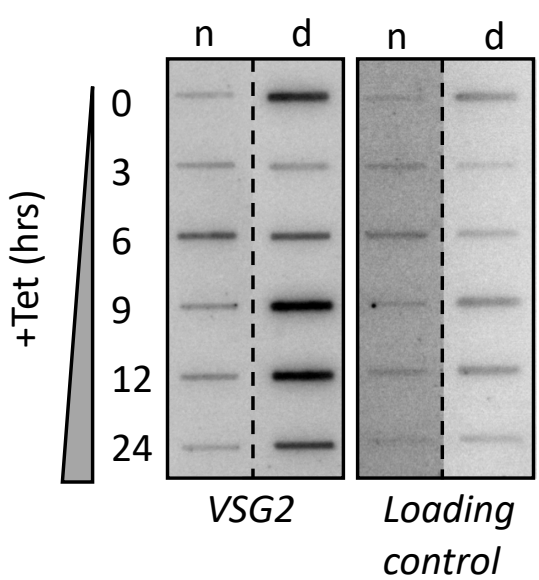

Figure 6: RAD50 is not required for VSG switching. (A) A schematic map shows the primer position at the active expression site. (B) Immunofluorescence assay for VSG2, showing the percentage of switched survivors in the VSG uprad50 cell line. (C) PCR analysis shows the percentage of switched survivors that kept ESAG1 and VSG2. $\mathrm{n}=24$ clones. Arrows indicate position of primers; box with diagonal lines, 70-bp repeats; vertical lines, telomere. (D) Accumulation of ssDNA was monitored using slot-blots. Genomic DNA was extracted as indicated following I-Scel induction. Ninety percent

64 the sample was 'native' ( $\mathrm{n}$; ssDNA) and ten percent was denatured (d). Probe VSG2 and the loading control are described in the materials and methods. 
One subclone was found to be VSG2 negative by immunofluorescence, puromycin sensitive, and ESAG1 positive and VSG2 positive by PCR. This suggests that this single clone had switched by in situ transcriptional activation of another VSG-ES (Figure 6B and C and Supplementary Figure 3), whereas all other clones had undergone VSG switching by recombination. In the VSG ${ }^{\text {up }}$ mre11 cells, all the puromycin sensitive subclones had switched VSG and lost the VSG2 gene as seen by PCR analysis (Supplementary Figure $4 C$ ). 18 out of the 20 subclones had also retained ESAG1. As with the VSGup rad50 cells, these clones are presumed to have repaired by gene conversion using the 70bp repeats. We then assessed the formation of ssDNA and found that as in the ${ }^{1} \mathrm{HR}$ rad50 nulls, the formation of SSDNA was not abolished but seemed to accumulate earlier - here at 6 hours post DSB induction (Figure 6D). These data suggest that loss of RAD50 or MRE11 does not impair the cell's ability to undergo switching by DNA recombination.

RAD50 promotes recombination using long stretches of homology.

Increased survival of the VSG ${ }^{\text {up }}$ rad50 nulls after induction of a DSB suggested a hyperrecombinogenic mechanism in this locus, through which the cells are able to repair and switch at a higher rate than in the parental cell line. One explanation for such enhanced repair could be through greater access to the silent VSG repertoire. In the T. brucei genome there are in excess of 2000 VSG genes found at the subtelomeric arrays ${ }^{1613,15,58}, 90 \%$ of which are associated with a stretch of 70 -bp repeat sequence $^{14}$ that can be used for homology during repair ${ }^{9}$. To ask if differences in VSG repertoire access explains increased survival of VSG ${ }^{\text {up }}$ rad50 cells after DSB induction, we used VSGseq ${ }^{10}$. In the VSG ${ }^{\text {up }}$ WT cell line, 83 VSG gene transcripts were significantly enriched in the induced cells compared with uninduced (Figure 7A) (greater than $2 \log _{2}$ fold change and $p$ value of less than 0.05). In the VSG ${ }^{\text {up }}$ rad50 cell line, a greater number of VSGs were detected: here 225 VSG transcripts were significantly enriched after I-Scel induction (Figure 7A). To understand this increase, we then looked at the genomic position of the VSG cohorts. In the VSGup cells, we found that approximately equal numbers of enriched VSGs mapped to the VSG-ES and minichromosomes relative the megabase arrays, despite the much greater number of genes in the latter component of the archive (Figure 7B, Supplementary Figure 7A). These data appear consistent with VSG switching having followed a loose hierarchy, as previously published ${ }^{59}$, with telomeric VSG preferred as donors. In the VSG ${ }^{\text {up }}$ rad50 cell line, enriched VSG genes also mapped to the VSG-ESs, minichromosomes and megabase arrays, but a significantly higher proportion (67\% compared with $34 \%$ in WT) were from subtelomeric arrays (Figure 7B, Supplementary Figure 7A). This suggests that the VSG ${ }^{\text {up }}$ rad50 cells are able to access a greater proportion of the silent VSG archive for repair and VSG switching. To ask if increased VSG switching in the absence of RAD50 could be explained by changes in the mechanism of recombination, we looked at the length of homology used for repair. Using BLAST analysis, we queried the significantly enriched VSGs against the telomeric end of BES1, searching for regions of homology (Figure 7C and Supplementary Figure 7B). This analysis revealed that, compared with the VSG ${ }^{\text {up }}$ WT cells, VSG genes activated in the VSG ${ }^{\text {up }}$ rad50 cells shared shorter stretches of homology with the active VSG2 locus. 
A

VSGup
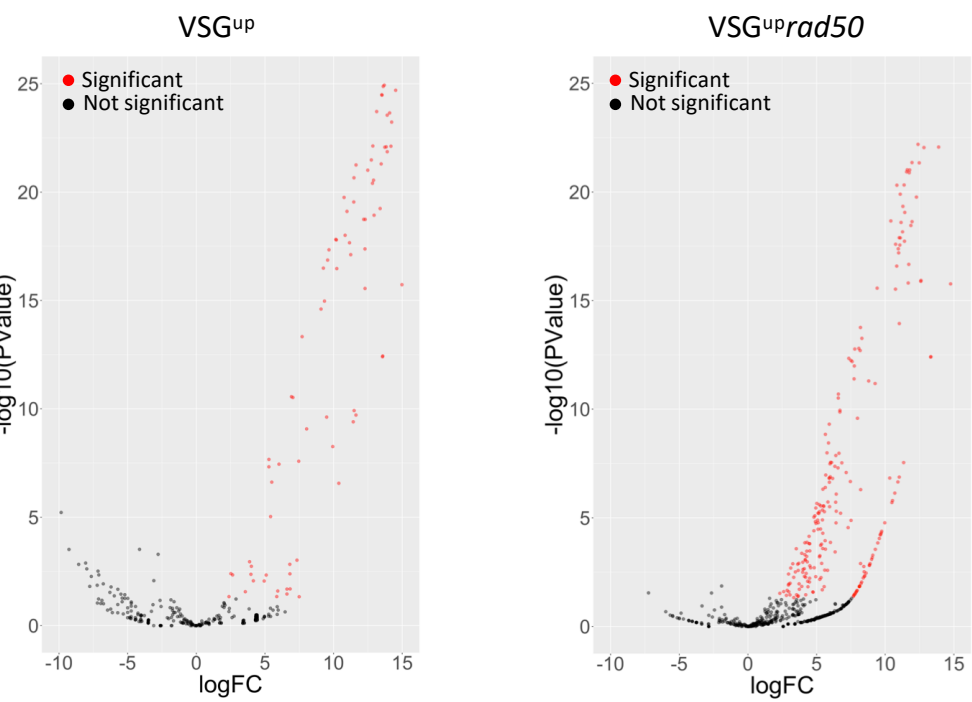

B

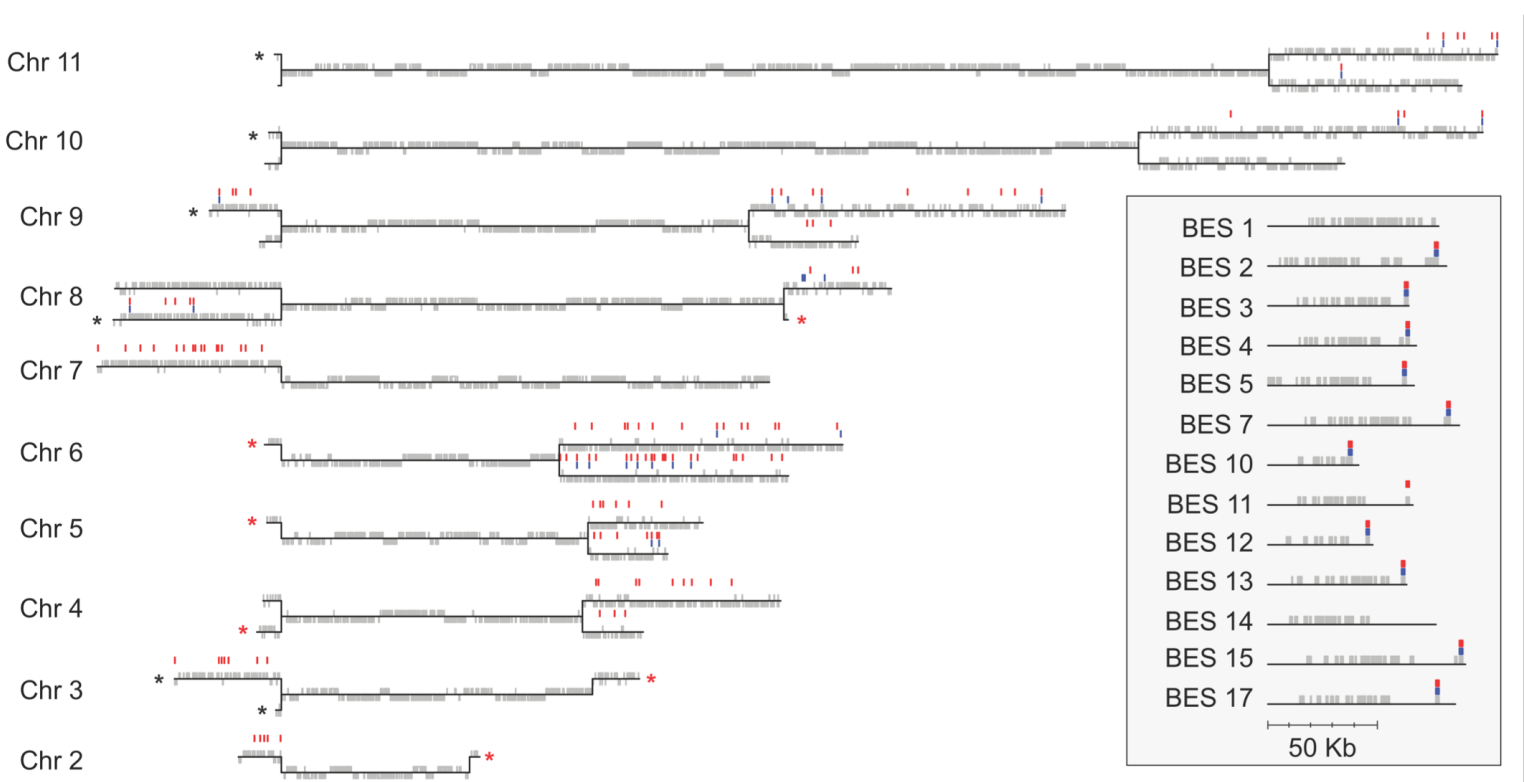

Chr 1

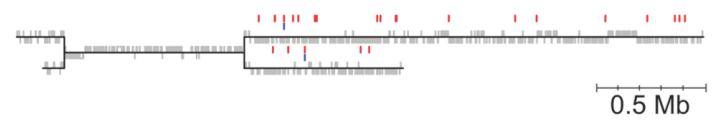

All genes

WT WSG LogFC > 2

rad50 1 VSG $P$ Value $<0.05$

* Bloodstream VSG-ES

C

* Metacyclic VSG-ES

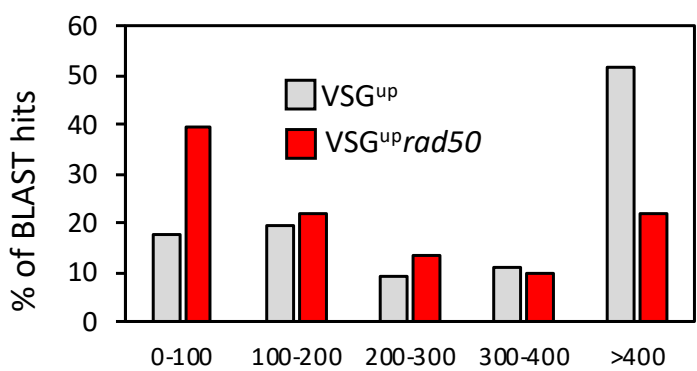

Length of BLAST hits (nucleotides)

508 Figure 7: RAD50 restricts antigenic variation. (A) Volcano plots of VSG-seq showing $\log _{2}$ fold change

509 vs. $\log _{10} P$ value for VSG ${ }^{\text {up }}$ and VSG ${ }^{\text {up }}$ rad50 strains. Red genes are significantly up-regulated ( $P$ value $<0.05$ and $\log _{2} \mathrm{FC}>2$.

(B) T. brucei 427 Genome map showing all 11 megabase chromosomes in 
black lines and all genes in grey. Significantly up-regulated VSG genes from either VSGup (blue) or VSG ${ }^{\text {up }}$ rad50 (red). Inset box shows all 13 BES with significantly up-regulated VSG genes from either VSG up (blue) or VSG ${ }^{\text {up }}$ rad50 (red). (C) Chromosome 6 map showing all genes in grey and significantly up-regulated VSG genes from either VSG ${ }^{\text {up }}$ (blue) or VSGup rad50 (red). (C) Percentage usage for the length of the BLAST hits from either VSG up (blue) or VSG ${ }^{\text {up }} R A D 50$ (red).Box with diagonal lines, 70-bp repeats; vertical lines, telomere; grey box, 3'UTR

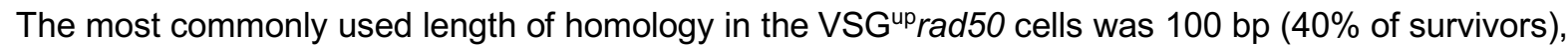
whereas the VSG up WT cells most frequently used $>400$ bp ( $51 \%$ of survivors) (Figure $7 C$ ). Thus, increased survival in the absence of RAD50 is due to increased access to archival VSGs due to more frequent DSB repair using short stretches of homology.

\section{DISCUSSION}

Central to the DDR and subsequent repair is the MRN complex, within which the MRE11 - RAD50 heterodimer forms the catalytic core. In fact, this core complex is so fundamental to repair that it is conserved across bacteriophages, bacteria, archaea and eukaryotes ${ }^{60}$. Kinetoplastids rely on homology-based recombination as the major form of repair, since no data has suggested the use of non-homologous end-joining after I-Scel induction of a DSB at both at chromosome-internal ${ }^{17}$ and telomeric loci in T. brucei, or after CRISPR-Cas9 DSB formation in Leishmania, T. cruzi or T. brucei ${ }^{61-}$ 63. At least in T. brucei, HR predominates over MMEJ after induction of a DSB ${ }^{17}$, and all evidence suggests RAD51-dependent HR dominates as the major form of repair reaction during VSG switching at the VSG-ES ${ }^{19}$. What dictates the preference of HR over MMEJ in these reactions in T. brucei and related kinetoplastids is unknown. Here, we have shown that $T$. brucei RAD50 is critical for normal cell growth and efficient homology-based DNA repair after induction of a DSB, which is consistent with a conserved role in DSB recognition and repair. We demonstrate that in the absence of RAD50 the DDR is severely compromised, as evidenced by the loss of $\gamma \mathrm{H} 2 \mathrm{~A}$ accumulation and the $\mathrm{G}_{2} / \mathrm{M}$ cell-cycle checkpoint at both subtelomeric and chromosome-internal DSBs, and the RPA focal accumulation. We also show that HR is dependent on RAD50, as null mutants displayed compromised RAD51 foci formation and switched repair pathway choice from HR to MMEJ at a chromosome-internal DSB. In contrast, at a VSG-ES we demonstrate that RAD50 restrains the DDR, since in its absence break processing dynamics were altered, cell survival improved and a greater diversity of VSGs were

543 activated following a DSB. Thus, this work provides insight into DSB repair pathway choice,

544 compartmentalization of repair with specific relevance for understanding trypanosome antigenic variation and genome maintenance in all kinetoplastids.

In T. brucei RAD51-dependent HR accounts for approximately 95\% of DSB repair at a chromosome-internal locus, and a DSB triggers a conventional DDR ${ }^{17}$. The data shown here indicate that the role of $T$. brucei RAD50 in recognizing a DSB and initiating the DDR appears consistent with its role in other eukaryotes ${ }^{64,65}$. Previous characterization of MRE11 has revealed a role in HR and genomic stability in both $T$. brucei ${ }^{46,47}$ and Leishmania ${ }^{49,66}$, suggesting RAD50 and MRE11 act 
together. Here we show that that MRE11 is essential for the DDR and HR and, in its absence, repair is predominantly achieved by MMEJ. We expect that the reduced survival of ${ }^{1} \mathrm{HRrad50}$ and VSG ${ }^{\mathrm{up}}$ rad50 cells is due to unrepaired DNA damage that results in cell death, which is also seen in mre11 mutants ${ }^{46,47}$. The persistence of RPA foci in the ${ }^{1} \mathrm{HRrad} 50$ cells is further evidence of defective repair in these cells, explained by RPA remaining associated with ssDNA at sites of damage. This observation may also reflect a damage tolerance strategy employed by $T$. brucei ${ }^{22}$, but in these circumstances $\gamma \mathrm{H} 2 \mathrm{~A}$ and RAD51 are not recruited to the break after replication, unlike what is seen in T. cruzi where ionizing radiation tolerance is dependent on RAD5167. DNA resection following a DSB is one of the early steps in the DDR. MRN is an early acting complex in DSB processing, initiating resection ${ }^{39,68}$ and recruiting the SGS2 nuclease and DNA2 and EXO1 for long-range resection thereafter ${ }^{39,69}$. The lack of resection defect in the ${ }^{1} \mathrm{HR}$ rad50 mutants, coupled with the altered dynamics of the formation and resolution of ssDNA, suggests that in trypanosomes there is flexibility in the nucleases able to initiate and resect across a DSB and, in the absence of MRN, unknown nucleases can act in an unrestricted manner that exceeds the resection rate of WT cells.

Loss of RAD50 in T. brucei reveals a striking difference in the response to a DSB at a chromosome-internal location and in the active VSG-ES, with dramatically impaired survival at the former and improved survival at the latter. This locus-specific effect of rad50 nulls was not reproduced in the mre11 nulls. It is possible that this difference arises from a shared change in DSB repair reaction mechanism due to loss of the MRN complex. After DSB induction, loss of RAD50 results in lower levels of $\mathrm{yH} 2 \mathrm{~A}$ and $\mathrm{RAD} 51$ foci, allied to more rapid formation and resolution of SSDNA at a chromosome-internal site, with the result that DSB repair no longer favours HR but instead mainly uses MMEJ. An explanation for these effects could be that loss of RAD50 leads to impaired DSB processing by MRE11, allowing altered resection that leads to lowered levels of RAD51 nucleoprotein filament formation and increased recruitment of the unknown $T$. brucei machinery for MMEJ. If the MMEJ reaction was more efficient than HR, the more rapid resolution of ssDNA would be explained. If this same redirection of DSB repair occurred in the VSG-ES, why might the difference in survival occur? A simple explanation might be that MMEJ repair is more error-prone (such as by mutation or translocation) than $\mathrm{HR}^{70}$, and the use of error-prone MMEJ has a greater impact in the chromosome core, where it would affect housekeeping genes, than in the subtelomeres, where there is a huge abundance of silent VSG substrates. Because the machinery that directs MMEJ in T. brucei or any kinetoplastid has not been characterised, directs tests of this suggestion are not yet possible. In addition, it remains to be shown that MMEJ directs the activation of silent array VSGs after a DSB in the VSG-ES, since the length of homologies we describe here (predominantly 100 bp) appear longer than the $\sim 5-15$ bp microhomologies so far detailed in experimental characterization of $T$. brucei MMEJ ${ }^{21,71}$. Nonetheless, previous work has shown considerable flexibility in the substrate requirements of recombination in $T$. brucei ${ }^{71}$, and linked MMEJ and HR-mediated repair of a DSB has been detailed ${ }^{21}$. Thus, it remains possible that MRN is pivotal in determining the routing of DNA repair throughout the $T$. brucei genome.

DNA DSBs are a potent trigger for $T$. brucei antigenic variation and it has been shown that the subtelomeric VSG-ES are fragile and prone to DSBs ${ }^{27}$. A DSB upstream of the active VSG triggers 
antigenic variation, and subsequent repair by recombination is facilitated by stretches of 70-bp repeat that provide homology ${ }^{9,26,27}$, via either a RAD51-dependent or independent pathway ${ }^{27}$. Given this, it remains perplexing that mutation of MRE11 was not previously shown to alter the rate of VSG switching ${ }^{46}$, unlike the clear alteration in survival and repair pathway seen here after loss of RAD50 and induction of a VSG-ES DSB. These data may suggest an unconventional signalling pathway following a DSB at the active VSG-ES that allows the cells to bypass the conventional MRN / ATM pathway. One way that might occur is that the DSBs in the VSG-ES do not arise directly, but are generated by routes such as clashes between replication and transcription ${ }^{31}$, or the formation of RNADNA hybrids ${ }^{72-74}$, which might not elicit recruitment of MRN or $\mathrm{ATM}^{75,76}$. Our data reveals that increased survival in the VSG ${ }^{\text {up }}$ rad50 cells is due to their ability to access more of the genomic VSG archive for repair, and the repair reaction occurs as a result of recombination using shorter stretches of homology. RAD50, therefore, appears to suppress antigenic variation by committing $T$. brucei to RAD51-dependent repair pathway that is reliant on long stretches of donor homology. We also note that we observe a striking number of donor VSG genes used for repair on Chromosome 6 - BES1, which contains the I-Scel site is found on Chromosome 6a. Indeed, several other factors have also been shown to suppress antigenic variation: $\mathrm{HAT}^{35}$, TOPO $3 \mathrm{a}^{32}$ and the telomere-interacting factor TIF $2^{77}$. Antigenic variation following a DSB is driven by break-induced repair ${ }^{26,27}$ and, in yeast, RAD51independent BIR has been shown require as little as $30 \mathrm{bp}$ of homology for repair ${ }^{78}$. Thus, loss of RAD50 may allow T. brucei to enlist RAD51-independent BIR to respond to a DSB in the VSG-ES, using shorter stretches of homology, rather than MMEJ. Irrespective, the question arises as to why $T$. brucei might limit the range of VSGs that can be used in antigenic variation, since this appears counter to the observed diversity of VSGs seen during infections ${ }^{10,11}$. It is possible that RAD50-mediated restraint of VSG recombination is needed to preserve the VSG archive, saving genes for use in prolonged infections. A more radical possibility is that RAD50/MRN control underlies the hierarchy of VSG expression, directing DSB repair to telomeric VSG substrates early an infection and then giving way to a distinctly signalled reaction, such as MMEJ, that allows access to the whole VSG archive later.

\section{DATA AVAILABILITY}

All script is hosted on GitHub : https://github.com/LGloverTMB/DNA-repair-mutant-VSG-seq All data has been deposited onto the ENA under study accession number: PRJEB37290 and unique study name: ena-STUDY-INSTITUT PASTEUR-15-03-2020-20:11:26:661-115

\section{ACKNOWLEDGEMENTS}


632 AK. M. was supported by the Erasmus + program of the European Union. Work in the L.G. laboratory 633 has received financial support from the Institut Pasteur and the National Research Agency (ANR 634 VSGREG). Aspects of the work in this grant was support by the Wellcome Trust $(089172,206815)$.

635 Further work in RM's lab was supported by the BBSRC (BB/K006495/1, BB/N016165/1), and the 636 Wellcome Centre for Integrative Parasitology is supported by core funds from the Wellcome Trust 637 (104111). S.H. is a Marie Curie fellow, this project has received funding from the European Union's 638 Horizon 2020 research and innovation programme under the Marie Skłodowska-Curie grant 639 agreement No 794979

640

6421 Trindade, S. et al. Trypanosoma brucei Parasites Occupy and Functionally Adapt to the 643 Adipose Tissue in Mice. Cell Host Microbe 19, 837-848, doi:10.1016/j.chom.2016.05.002 (2016). Capewell, P. et al. The skin is a significant but overlooked anatomical reservoir for vectorborne African trypanosomes. Elife 5, doi:10.7554/eLife.17716 (2016).

$6494 \quad$ Baltz, T. et al. Chemical and immunological characterization of specific glycoproteins from Trypanosoma equiperdum variants. FEBS Lett 82, 93-96, doi:10.1016/0014-5793(77)80893-4 (1977).

654 virulent and avirulent subspecies. Exp Parasitol 59, 98-107, doi:10.1016/00144894(85)90062-1 (1985).

656

6 Schwede, A., Jones, N., Engstler, M. \& Carrington, M. The VSG C-terminal domain is inaccessible to antibodies on live trypanosomes. Mol Biochem Parasitol 175, 201-204,

doi:10.1016/j.molbiopara.2010.11.004 (2011).

6587 Sima, N., McLaughlin, E. J., Hutchinson, S. \& Glover, L. Escaping the immune system by DNA repair and recombination in African trypanosomes. Open Biol 9, 190182, doi:10.1098/rsob.190182 (2019).

6618 Hertz-Fowler, C. et al. Telomeric expression sites are highly conserved in Trypanosoma $662 \quad$ brucei. PLoS One 3, e3527, doi:10.1371/journal.pone.0003527 (2008).

6639 Hovel-Miner, G., Mugnier, M. R., Goldwater, B., Cross, G. A. \& Papavasiliou, F. N. A 664 Conserved DNA Repeat Promotes Selection of a Diverse Repertoire of Trypanosoma brucei 665 Surface Antigens from the Genomic Archive. PLoS Genet 12, e1005994, doi:10.1371/journal.pgen.1005994 (2016).

66710 Mugnier, M. R., Cross, G. A. \& Papavasiliou, F. N. The in vivo dynamics of antigenic variation 668 in Trypanosoma brucei. Science 347, 1470-1473, doi:10.1126/science.aaa4502 (2015).

66911 Hall, J. P., Wang, H. \& Barry, J. D. Mosaic VSGs and the scale of Trypanosoma brucei 670 antigenic variation. PLoS Pathog 9, e1003502, doi:10.1371/journal.ppat.1003502 (2013). 
Jayaraman, S. et al. Application of long read sequencing to determine expressed antigen diversity in Trypanosoma brucei infections. PLoS Negl Trop Dis 13, e0007262, doi:10.1371/journal.pntd.0007262 (2019). (the VSGnome) of Trypanosoma brucei Lister 427. Mol Biochem Parasitol 195, 59-73, doi:10.1016/j.molbiopara.2014.06.004 (2014). Marcello, L. \& Barry, J. D. Analysis of the VSG gene silent archive in Trypanosoma brucei reveals that mosaic gene expression is prominent in antigenic variation and is favored by archive substructure. Genome Res 17, 1344-1352, doi:10.1101/gr.6421207 (2007). Muller, L. S. M. et al. Genome organization and DNA accessibility control antigenic variation in trypanosomes. Nature 563, 121-125, doi:10.1038/s41586-018-0619-8 (2018). 309, 416-422, doi:10.1126/science.1112642 (2005). chromosomal double-strand break repair in African trypanosomes. Nucleic Acids Res 36, 2608-2618, doi:10.1093/nar/gkn104 (2008). Trypanosoma brucei antigenic variation. Genes Dev 13, 2875-2888 (1999). gene conversion in antigenic variation in African trypanosomes. Mol Cell Biol 19, 5839-5846 (1999). homologous recombination in Trypanosoma brucei. Mol Microbiol 45, 1687-1700, doi:10.1046/j.1365-2958.2002.03122.x (2002).

704 African trypanosomes. Nucleic Acids Res 39, 1372-1380, doi:10.1093/nar/gkq981 (2011). Glover, L., Marques, C. A., Suska, O. \& Horn, D. Persistent DNA Damage Foci and DNA Replication with a Broken Chromosome in the African Trypanosome. MBio 10, doi:10.1128/mBio.01252-19 (2019). independent end joining in Trypanosoma brucei cell extracts relies upon sequence microhomology. Eukaryot Cell 6, 1773-1781, doi:10.1128/EC.00212-07 (2007).

4 Nenarokova, A. et al. Causes and Effects of Loss of Classical Nonhomologous End Joining Pathway in Parasitic Eukaryotes. MBio 10, doi:10.1128/mBio.01541-19 (2019). 5 Shibata, A. Regulation of repair pathway choice at two-ended DNA double-strand breaks. Mutat Res 803-805, 51-55, doi:10.1016/j.mrfmmm.2017.07.011 (2017).

26 Boothroyd, C. E. et al. A yeast-endonuclease-generated DNA break induces antigenic switching in Trypanosoma brucei. Nature 459, 278-281, doi:10.1038/nature07982 (2009). 
Glover, L., Alsford, S. \& Horn, D. DNA break site at fragile subtelomeres determines probability and mechanism of antigenic variation in African trypanosomes. PLoS Pathog $\mathbf{9}$, e1003260, doi:10.1371/journal.ppat.1003260 (2013).

Proudfoot, C. \& McCulloch, R. Distinct roles for two RAD51-related genes in Trypanosoma brucei antigenic variation. Nucleic Acids Res 33, 6906-6919, doi:10.1093/nar/gki996 (2005). Trenaman, A. et al. Trypanosoma brucei BRCA2 acts in a life cycle-specific genome stability process and dictates BRC repeat number-dependent RAD51 subnuclear dynamics. Nucleic Acids Res 41, 943-960, doi:10.1093/nar/gks1192 (2013). Hartley, C. L. \& McCulloch, R. Trypanosoma brucei BRCA2 acts in antigenic variation and has undergone a recent expansion in $\mathrm{BRC}$ repeat number that is important during homologous recombination. Mol Microbio/ 68, 1237-1251, doi:10.1111/j.1365-2958.2008.06230.x (2008). Devlin, R. et al. Mapping replication dynamics in Trypanosoma brucei reveals a link with telomere transcription and antigenic variation. Elife 5, doi:10.7554/eLife.12765 (2016). $\mathrm{Kim}, \mathrm{H}$. S. \& Cross, G. A. TOPO3alpha influences antigenic variation by monitoring expression-site-associated VSG switching in Trypanosoma brucei. PLoS Pathog 6, e1000992, doi:10.1371/journal.ppat.1000992 (2010).

Kim, H. S. \& Cross, G. A. Identification of Trypanosoma brucei RMI1/BLAP75 homologue and its roles in antigenic variation. PLoS One 6, e25313, doi:10.1371/journal.pone.0025313 (2011). Variation with Regulation of RNA Polymerase I-Transcribed Surface Antigens. Cell Rep 30, 836-851 e835, doi:10.1016/j.celrep.2019.12.049 (2020). Glover, L. \& Horn, D. Locus-specific control of DNA resection and suppression of subtelomeric VSG recombination by HAT3 in the African trypanosome. Nucleic Acids Res 42, 1260012613, doi:10.1093/nar/gku900 (2014). Ciccia, A. \& Elledge, S. J. The DNA damage response: making it safe to play with knives. Mol Cell 40, 179-204, doi:10.1016/j.molcel.2010.09.019 (2010). Syed, A. \& Tainer, J. A. The MRE11-RAD50-NBS1 Complex Conducts the Orchestration of Damage Signaling and Outcomes to Stress in DNA Replication and Repair. Annu Rev Biochem 87, 263-294, doi:10.1146/annurev-biochem-062917-012415 (2018). Lisby, M., Barlow, J. H., Burgess, R. C. \& Rothstein, R. Choreography of the DNA damage response: spatiotemporal relationships among checkpoint and repair proteins. Cell 118, 699713, doi:10.1016/j.cell.2004.08.015 (2004).

74641 Zhang, Y., Zhou, J. \& Lim, C. U. The role of NBS1 in DNA double strand break repair, telomere stability, and cell cycle checkpoint control. Cell Res 16, 45-54, doi:10.1038/sj.cr.7310007 (2006). 

resection. Acta Biochim Biophys Sin (Shanghai) 48, 647-657, doi:10.1093/abbs/gmw043 (2016). DNA double-strand break repair and the ABC-ATPase superfamily. Cell 101, 789-800, doi:10.1016/s0092-8674(00)80890-9 (2000). (Basel) 9, doi:10.3390/genes9120589 (2018). Dev 22, 125-140, doi:10.1101/gad.1626908 (2008). Robinson, N. P., McCulloch, R., Conway, C., Browitt, A. \& Barry, J. D. Inactivation of Mre11 does not affect VSG gene duplication mediated by homologous recombination in Trypanosoma brucei. J Biol Chem 277, 26185-26193, doi:10.1074/jbc.M203205200 (2002). influences growth, homologous recombination and DNA double-strand break repair. Mol Biochem Parasitol 125, 11-21 (2002). Hopfner, K. P. et al. Structural biochemistry and interaction architecture of the DNA doublestrand break repair Mre11 nuclease and Rad50-ATPase. Cell 105, 473-485 (2001). Laffitte, M. C. et al. Chromosomal Translocations in the Parasite Leishmania by a MRE11/RAD50-Independent Microhomology-Mediated End Joining Mechanism. PLoS Genet 12, e1006117, doi:10.1371/journal.pgen.1006117 (2016). recombination and repair. Nature 418, 562-566, doi:10.1038/nature00922 (2002). Williams, R. S., Williams, J. S. \& Tainer, J. A. Mre11-Rad50-Nbs1 is a keystone complex connecting DNA repair machinery, double-strand break signaling, and the chromatin template. Biochem Cell Biol 85, 509-520, doi:10.1139/O07-069 (2007). critical to ATP hydrolysis and cooperativity in bacteriophage T4 Rad50. J Biol Chem 286, 26258-26266, doi:10.1074/jbc.M111.256305 (2011). for protein modeling, prediction and analysis. Nat Protoc 10, 845-858, doi:10.1038/nprot.2015.053 (2015).

Jackson, S. P. \& Bartek, J. The DNA-damage response in human biology and disease. Nature 461, 1071-1078, doi:10.1038/nature08467 (2009). Biochem Parasitol 183, 78-83, doi:10.1016/j.molbiopara.2012.01.008 (2012).

Stauffer, M. E. \& Chazin, W. J. Physical interaction between replication protein A and Rad51 promotes exchange on single-stranded DNA. J Biol Chem 279, 25638-25645, doi:10.1074/jbc.M400029200 (2004). 
Ingram, A. K., Cross, G. A. \& Horn, D. Genetic manipulation indicates that ARD1 is an essential N(alpha)-acetyltransferase in Trypanosoma brucei. Mol Biochem Parasitol 111, 309317 (2000).

Glover, L. et al. Antigenic variation in African trypanosomes: the importance of chromosomal and nuclear context in VSG expression control. Cell Microbiol 15, 1984-1993, doi:10.1111/cmi.12215 (2013). Trypanosoma brucei: joining the DOTs. PLoS Bio/ 6, e185, doi:10.1371/journal.pbio.0060185 (2008). Rad50 complex. Trends Biochem Sci 27, 410-418 (2002). targeted gene editing and precision base editing in African trypanosomes. Sci Rep 8, 7960, doi:10.1038/s41598-018-26303-w (2018). Waldrip, Z. J. et al. A CRISPR-based approach for proteomic analysis of a single genomic locus. Epigenetics 9, 1207-1211, doi:10.4161/epi.29919 (2014). Zhang, W. W. \& Matlashewski, G. CRISPR-Cas9-Mediated Genome Editing in Leishmania donovani. MBio 6, doi:10.1128/mBio.00861-15 (2015). breaks requires the Mre11/Rad50/Xrs2 complex. Nat Cell Biol 3, 844-847, doi:10.1038/ncb0901-844 (2001).

Marechal, A. \& Zou, L. DNA damage sensing by the ATM and ATR kinases. Cold Spring Harb Perspect Biol 5, doi:10.1101/cshperspect.a012716 (2013). Laffitte, M. C. et al. Formation of linear amplicons with inverted duplications in Leishmania requires the MRE11 nuclease. PLoS Genet 10, e1004805, doi:10.1371/journal.pgen.1004805 (2014). the repair of DNA double strand breaks and oxidative lesions. PLoS Negl Trop Dis 12, e0006875, doi:10.1371/journal.pntd.0006875 (2018). recombinational repair at random, gamma-induced double-strand break ends. PLoS Genet $\mathbf{5}$, e1000656, doi:10.1371/journal.pgen.1000656 (2009).

Zhu, Z., Chung, W. H., Shim, E. Y., Lee, S. E. \& Ira, G. Sgs1 helicase and two nucleases Dna2 and Exo1 resect DNA double-strand break ends. Cell 134, 981-994, doi:10.1016/j.cell.2008.08.037 (2008).

Seol, J. H., Shim, E. Y. \& Lee, S. E. Microhomology-mediated end joining: Good, bad and ugly. Mutat Res 809, 81-87, doi:10.1016/j.mrfmmm.2017.07.002 (2018). 
repair as substrate length decreases. Nucleic Acids Res 35, 3478-3493, doi:10.1093/nar/gkm249 (2007).

82972 Briggs, E., Crouch, K., Lemgruber, L., Lapsley, C. \& McCulloch, R. Ribonuclease H1-targeted

830 R-loops in surface antigen gene expression sites can direct trypanosome immune evasion. PLoS Genet 14, e1007729, doi:10.1371/journal.pgen.1007729 (2018).

$83273 \quad$ Briggs, E. et al. Trypanosoma brucei ribonuclease H2A is an essential R-loop processing enzyme whose loss causes DNA damage during transcription initiation and antigenic variation. Nucleic Acids Res, doi:10.1093/nar/gkz644 (2019).

835 Chang, E. Y. et al. MRE11-RAD50-NBS1 promotes Fanconi Anemia R-loop suppression at transcription-replication conflicts. Nat Commun 10, 4265, doi:10.1038/s41467-019-12271-w (2019).

839 Mouse Model. Mol Cancer Res 14, 185-195, doi:10.1158/1541-7786.MCR-15-0281 (2016). replication fork degradation by MRE11. Cell 145, 529-542, doi:10.1016/j.cell.2011.03.041 (2011). Using Overlapping and Independent Mechanisms. PLoS One 11, e0156746, doi:10.1371/journal.pone.0156746 (2016). Ira, G. \& Haber, J. E. Characterization of RAD51-independent break-induced replication that acts preferentially with short homologous sequences. Mol Cell Biol 22, 6384-6392, doi:10.1128/mcb.22.18.6384-6392.2002 (2002).

855 Hirumi, H. \& Hirumi, K. Continuous cultivation of Trypanosoma brucei blood stream forms in a medium containing a low concentration of serum protein without feeder cell layers. $J$ Parasitol 75, 985-989 (1989). van den Hoff, M. J., Moorman, A. F. \& Lamers, W. H. Electroporation in 'intracellular' buffer increases cell survival. Nucleic Acids Res 20, 2902 (1992).

81 Jeacock, L., Baker, N., Wiedemar, N., Maser, P. \& Horn, D. Aquaglyceroporin-null

$86384 \quad$ Devlin, R. et al. Mapping replication dynamics in Trypanosoma brucei reveals a link with 864 trypanosomes display glycerol transport defects and respiratory-inhibitor sensitivity. PLoS Pathog 13, e1006307, doi:10.1371/journal.ppat.1006307 (2017).

Alsford, S. \& Horn, D. Single-locus targeting constructs for reliable regulated RNAi and transgene expression in Trypanosoma brucei. Mol Biochem Parasitol 161, 76-79, doi:10.1016/j.molbiopara.2008.05.006 (2008).

83 Alsford, S. \& Horn, D. Single-locus targeting constructs for reliable regulated RNAi and transgene expression in Trypanosoma brucei. Mol Biochem Parasitol 161, 76-79, doi:10.1016/j.molbiopara.2008.05.006 (2008). telomere transcription and antigenic variation. eLife 5, e12765, doi:10.7554/eLife.12765 (2016). 

histone acetylation. Mutation research 750, 23-30, doi:10.1016/j.mrfmmm.2013.07.002 (2013). Woodward, R. \& Gull, K. Timing of nuclear and kinetoplast DNA replication and early morphological events in the cell cycle of Trypanosoma brucei. J Cell Sci 95 ( Pt 1), 49-57 (1990). 357-359, doi:10.1038/nmeth.1923 (2012). 2079, doi:10.1093/bioinformatics/btp352 (2009). assigning sequence reads to genomic features. Bioinformatics 30, 923-930, doi:10.1093/bioinformatics/btt656 (2014).

\section{TABLE AND SUPPLEMENTARY FIGURE LEGENDS}

884

Supplementary figure 1: Sequence alignment of the T. brucei RAD50 (TbRAD50) with Homo sapiens (HsRAD50; UNIPROT: Q92878). Conserved domains are highlighted in bold and the ABC/ATPase domains highlighted with grey bar. Highly conserved residues are highlighted with a * and residues with conserved properties with ':' or '. .

Supplementary figure 2: Generation of rad50 and mre11 null strains. (A) Upper panel: Schematic showing the knockout strategy to generate the chromosome-internal ${ }^{1} \mathrm{HR}$ rad50 null cell line. (B) PCR assay confirming RAD50 double allele replacement. (C) PCR assay confirming MRE11 double allele replacement. * background band. NEO, Neomycin Phosphotransferase; BLA, Blasticidin deaminase. C1, control plasmid for BLA; C2, control plasmid for NEO.

Supplementary figure 3: Resection is compromised in the ${ }^{1} \mathrm{HRrad50}$ nulls Left panel: Biological replicate showing accumulation of ssDNA was monitored using slot-blots, $\mathrm{n}=2$ for biological replicates. Right panel: Quantitative analysis the amount of ssDNA. Error bars, SD for biological replicates for the rad50 null strains; $\mathrm{n}=2$.

Supplementary figure 4: MRE11 is essential for DSB response and repair at a chromosome-internal locus. (A) Clonogenic assay reveals survivors following a DSB at a chromosome-internal locus in the parental and ${ }^{1} \mathrm{HRmre} 11$ null cell lines. Details as in Figure $1{ }^{1} \mathrm{HR}$ technical replicates; $\mathrm{n}=2$, and with monitoring $\gamma \mathrm{H} 2 \mathrm{~A}$ foci. The number of positive nuclei were counted in uninduced cells and 12 hours 
post DSB. Middle panel: Immunofluorescence assay to monitoring RAD51 foci. The number of positive nuclei were counted in uninduced cells and 12 hours post DSB. Lower panel: The number of cells in G2/M phase cells was counted by DAPI staining at several points following induction of an I-Scel break in. $\mathrm{G} 2$ cells contain one nucleus and two kinetoplasts. Error bars, SD, for ${ }^{1} \mathrm{HRmre} 11$ biological replicates for the strains; $n=2$.

(C) PCR analysis of repaired subclones. Upper panel: Schematic showing the 2110 locus and position of the Sce recognition site $\left(\mathrm{Sce}^{\mathrm{R}}\right)$. Position of primers indicated by arrows. Primer sequence detailed in materials and methods. Middle panel: PCR assay of repaired subclones showing RFP:PAC presence or absence. Lower panel: PCR assay of repaired subclones that were negative for RFP:PAC. Arrows indicate position of primers. White box, genes; Grey box, RFP:PAC fusion gene; black box, UTRs.

Supplementary figure 5: Analysis of VSG ${ }^{\text {up } R A D 50}$ strains. (A) Schematic showing the telomeric end of the VSG2 BES. (B) Presence or absence of ESAG1 in the repaired subclones. (C) Presence or absence of VSG2 in the repaired subclones. Arrows indicate position of primers; box with diagonal lines, 70-bp repeats; white boxes, genes; $\psi$, VSG pseudo gene; vertical lines, telomere.

Supplementary figure 6: MRE11 is essential for DSB response and repair at an expression site. (A) Clonogenic assay reveals survivors following a DSB in the VSG up strain in the parental and VSGUP mre11 cell lines. Details as in Figure 1 (B) Upper panel: Immunofluorescence assay to monitoring $\gamma \mathrm{H} 2 \mathrm{~A}$ foci. The number of positive nuclei were counted in uninduced cells and 12 hours post DSB. Lower panel: The number of cells in G2/M phase cells was counted by DAPI staining at several points following induction of an I-Scel break in. G2 cells contain one nucleus and two kinetoplasts. Error bars, SD, for VSG ${ }^{\mathrm{up}} m r e 11$ biological replicates for the strains; $n=2$. (C) PCR analysis of repaired subclones. Upper panel: Schematic showing BES1. Position of primers indicated by arrows. Primer sequence detailed in materials and methods. Middle panel: PCR assay of repaired subclones showing ESAG1 presence or absence. Lower panel: PCR assay of repaired subclones that were negative for VSG2 by immunofluorescence. Arrows indicate position of primers. White box, genes; ; $\psi$, pseudo gene; lined box, 70 bp repeats; black box, UTRs. VSG ${ }^{\text {up } r m r e 11 ~ b i o l o g i c a l ~}$ replicates for the strains; $n=2$.

Supplementary figure 7: (A) Table showing position of the VSG genes used for recombination (C) Proportion of VSG2 in the populations before and after induction of a DSB. (D) Schematic represents the telomeric end of the VSG2 ES. BLAST analysis of significantly up-regulated genes. BLAST hits are represented as lines showing their position on the VSG2 query sequence. Black lines - VSG genes significantly enriched in both VSG up and VSG up rad50, blue bars - VSG up only, red bars VSGup rad50 only. 


\title{
Supplementary Figure 1
}

HsRAD 50

TbRAD 50

HsRAD 50

TbRAD 50

Hs RAD 50

TbRAD 50

HsRAD 50

TbRAD 50

HsRAD 50

TbRAD 50

Hs RAD 50

TbRAD 50

HSRAD 50

TbRAD 50

Hs RAD 50

TbRAD 50

HsRAD 50

TBRAD 50

Hs RAD 50

TbRAD 50

\begin{abstract}
Walker A/P-loop

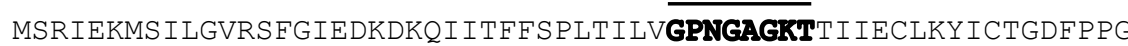

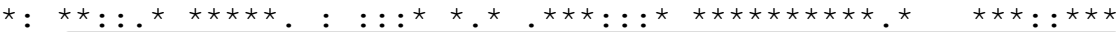
ABC RAD50 N
\end{abstract}

60

60

T--KGNTFVHDPKVAQETDVRAQIRLQFRDVNGELIAVQRSMVCTQKSKKTEFKTLEGVI GGTEKSSFVYDPKVVGENDVKAQIRLLFTGRGGKVMQVIRSFQATRTRNKTTFATLDNIV

118

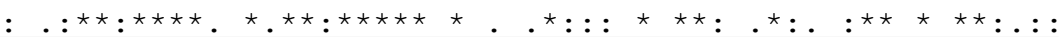

\section{Q-loop/lid}

TRT--KHGEKVSLSSKCAEIDREMISSLGVSKAVLNNV $\overline{\text { IFCH}} Q E D S N W P L S E G K A L K Q K F$ AFQDSATGKI ISSTYRANDVDRAI PDMLGVSPAVLEHVIFCHQEDGNWPLSP PKEVKKIF

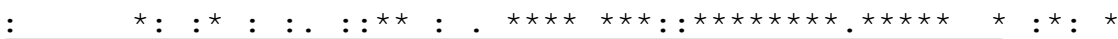

MRE11 binding site

DEIFSATRYIKALETLRQVRQTQGQKVKEYQMELKYLKQYKEKACEIRDQITSKEAQLTS DDIFAATRYVLALDRLRENNKELRRQQKEHEASLMSLSEHREQARQISADITVKEETVAG

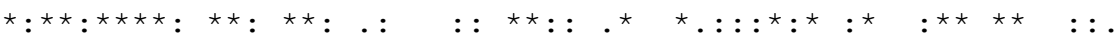

\section{Zn hook}

IEKSSKQRAMLAGAT---AVYSQF ITQLTDENQS CCPVC QRVFQTEAELQEVISDLQSKL RDRYHKLNEKLSGSKALAACHAHFVEQ--AKVEDKCPLCGRAFGSENELNDFLASFKVGQ

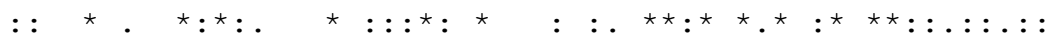

KLEENIDNIKRNHNLALGRQKGYEEEI I HFKKELREPQFRDAEEKYREMMIVMRTTELV $\overline{\mathbf{N}}$ LIRGKISALECLRAQQDGVAEAMRQDIESLKGQLTRDKYKDIEKRYRTTFLKVQTTEIAV

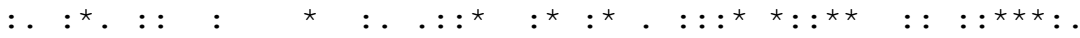

MRE11 binding site

KDLDIYYKTLDQAIMKFHSMKMEE INKI IRDLWRSTYRGQDIEYIEIRSDADENVSASDK SDVEKYYRALEKAVQTYHQEKIAQ INQ I LADLWRHTYKGSDIDTIELRSEDD--VTSTTA

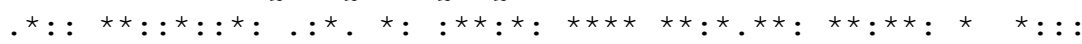

$A B C$ transporter signature motif

Walker B D-loop RRNYNYRVVMLKGDTALDMRGRCSAGQKVLAS L I IRLALAET FCLNCG $\overline{\text { IIALDE PTTNLD }}$ RRSYSYRVVMKRGNSEMDMRGRCSAGQKVLASVLIRLALSEAFCCDCGILALDEPTTNLD

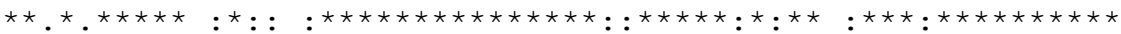

\section{$\mathrm{H}$-loop/switch region}

RENIESLAHALVEI IKSRSQQRNFQLLVITHDEDFVELLGRSEYVEKFYRIKKNIDQCSE EDNARS LAES LRMLIDSHRAVKHFQLIVITHDEHFVRALGGQALDT-FYYIHKDREGAFS

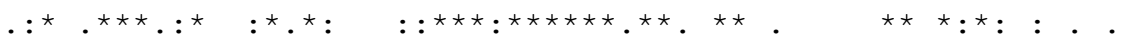

\section{ABC RAD50 C}

$\begin{array}{ll}\text { IVKCSVSSLGFNVH } & 1312 \\ \text { VIEERTFDQLFAS- } & 1349\end{array}$

1349 


\section{Supplementary Figure 2}

A

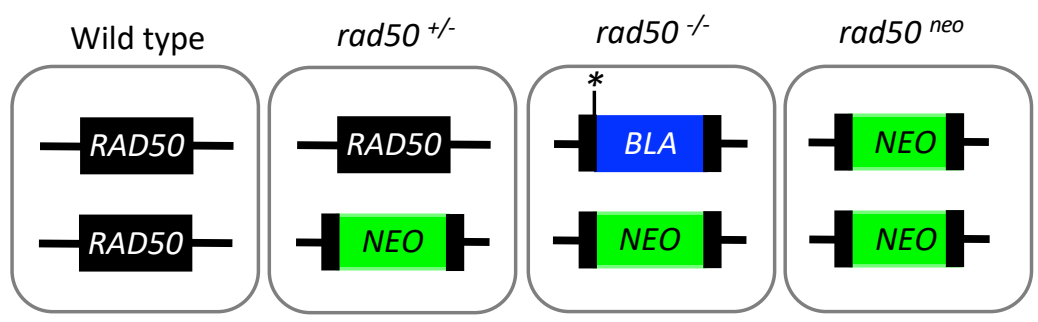

B

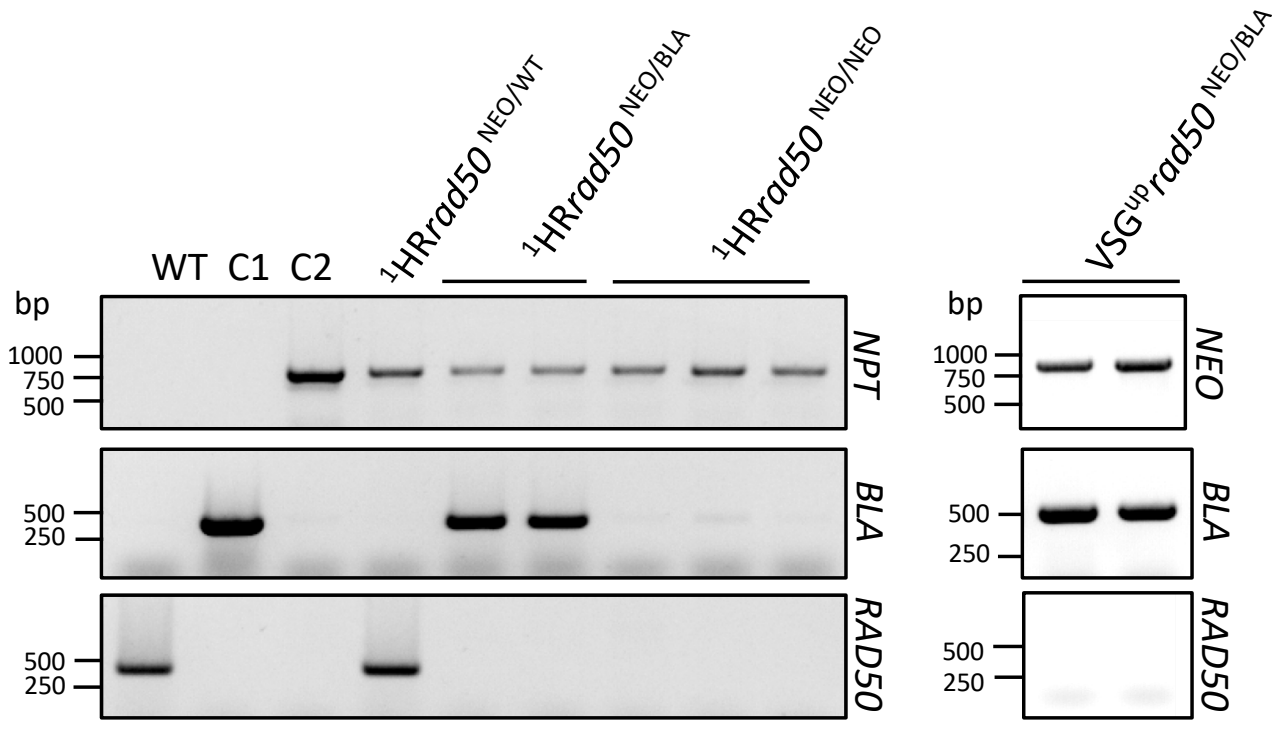

C

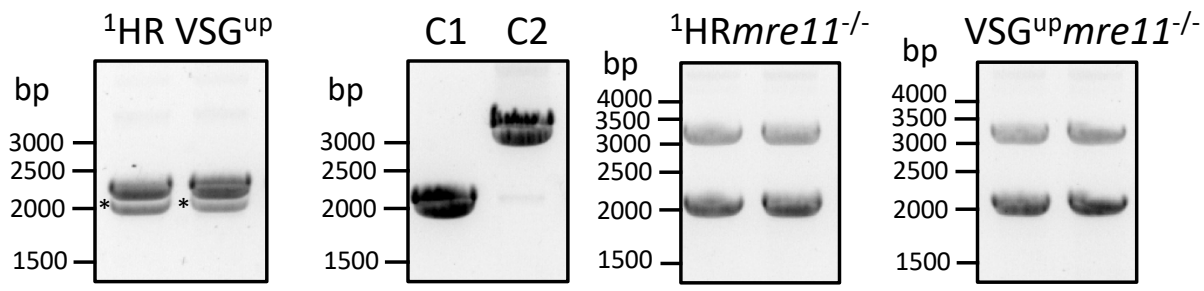



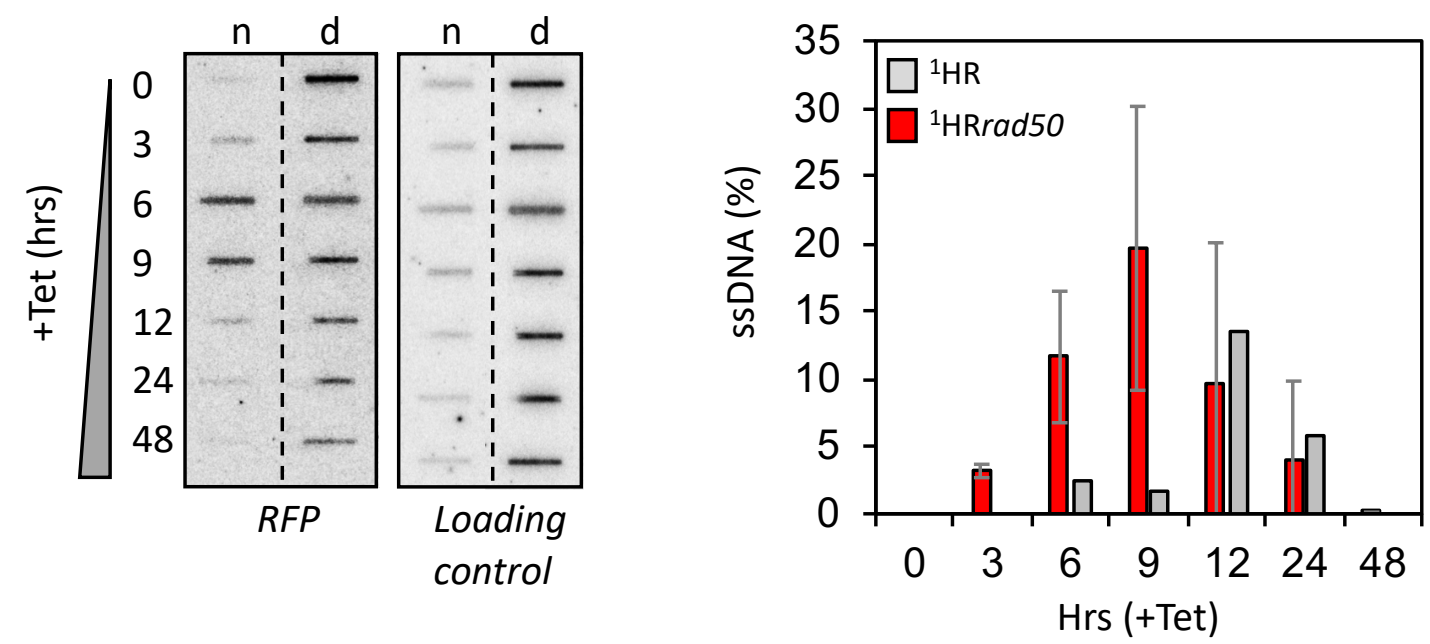
A

125

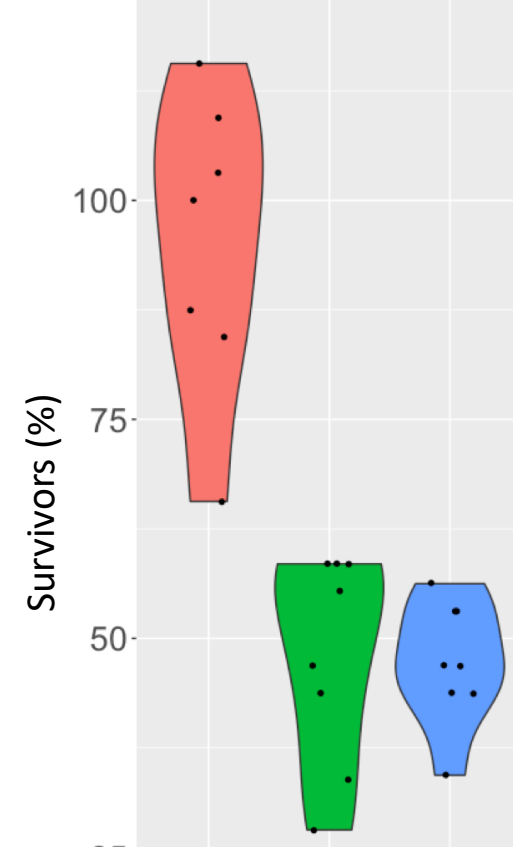

25

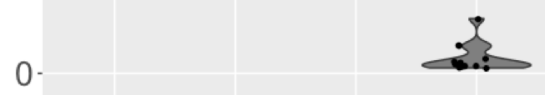

0.00

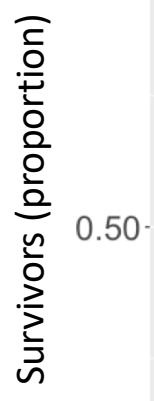

0.25
B
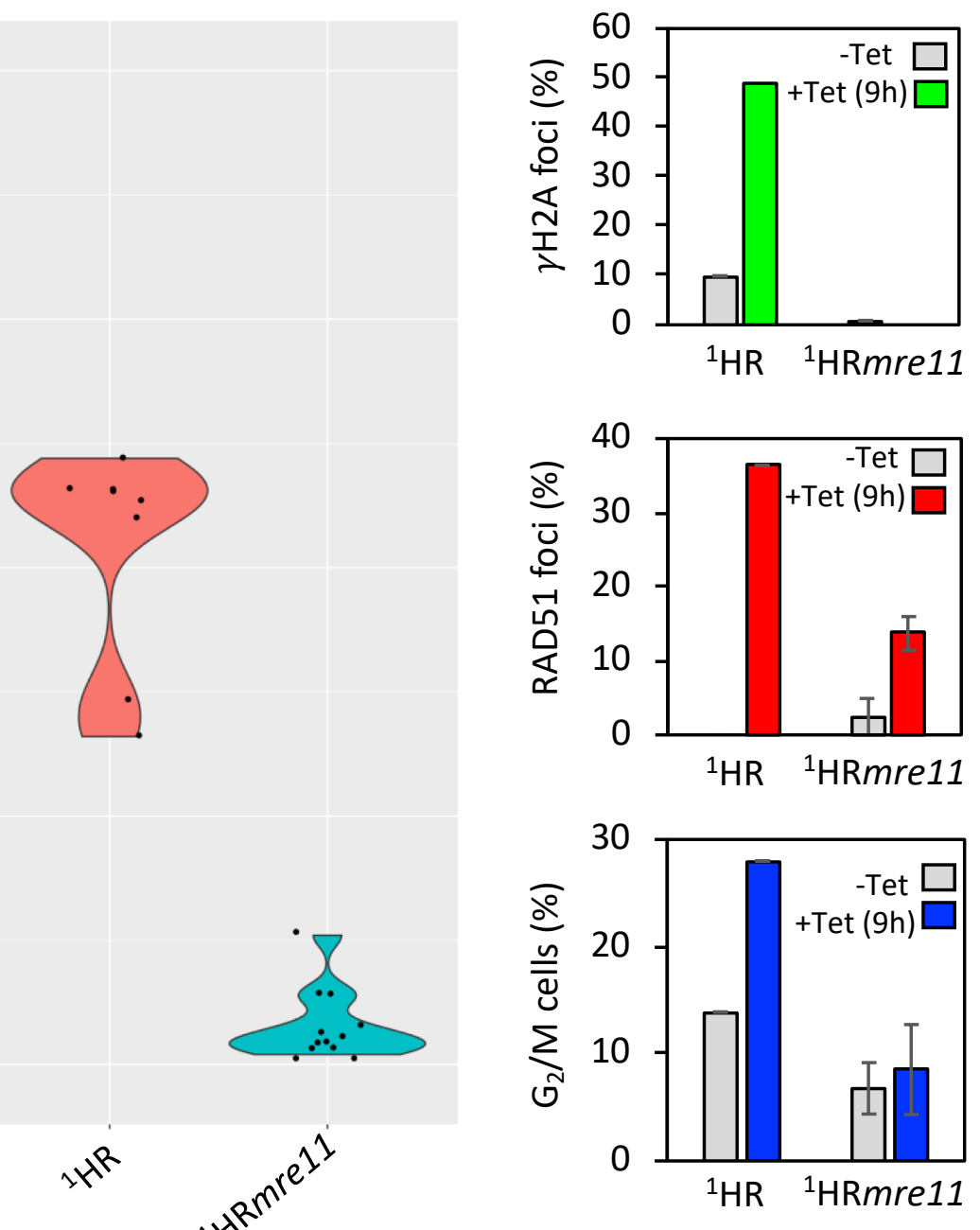

C
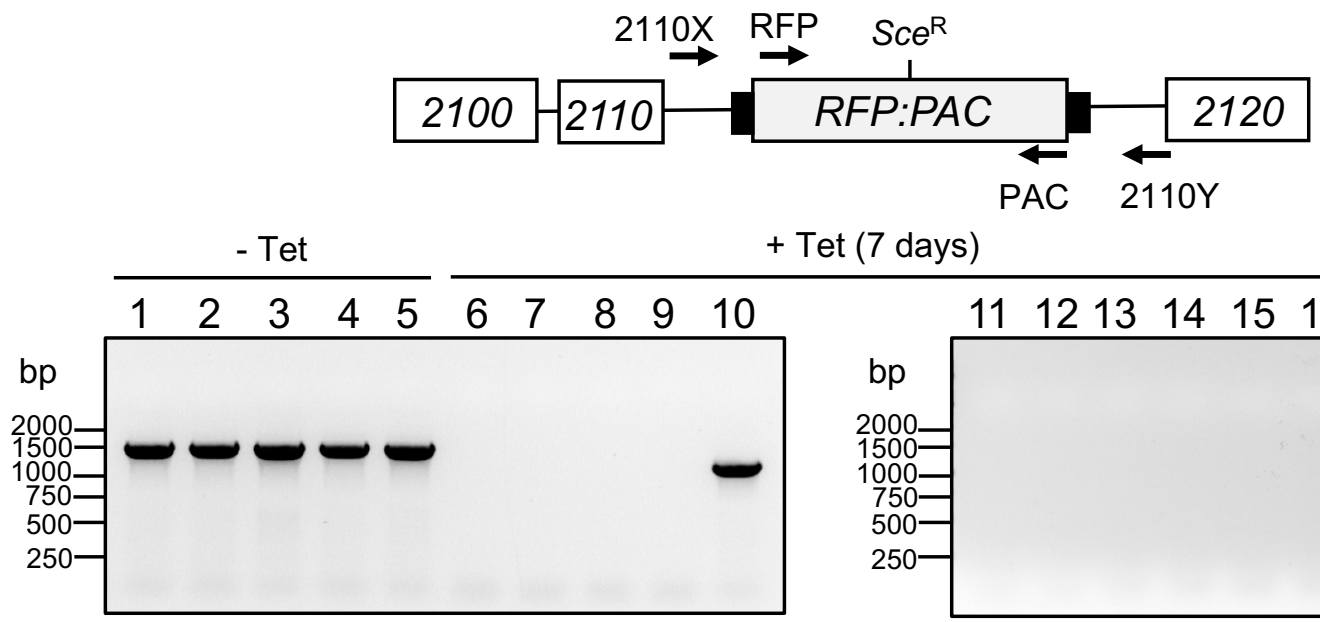

+ Tet (7 days)

RFP-PAC-ve clones
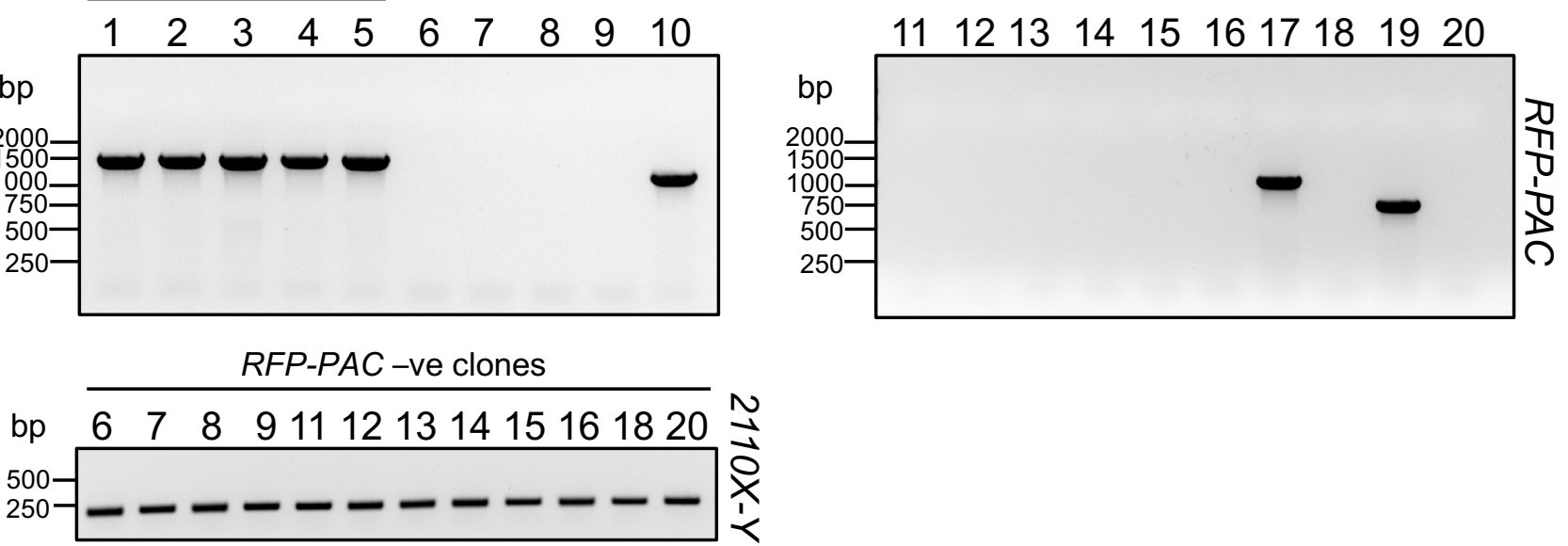


\section{Supplementary Figure 5}

A

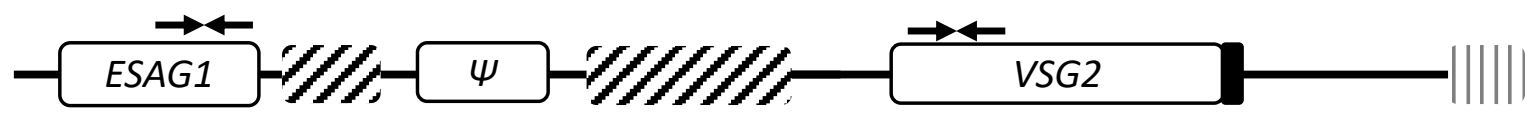

B

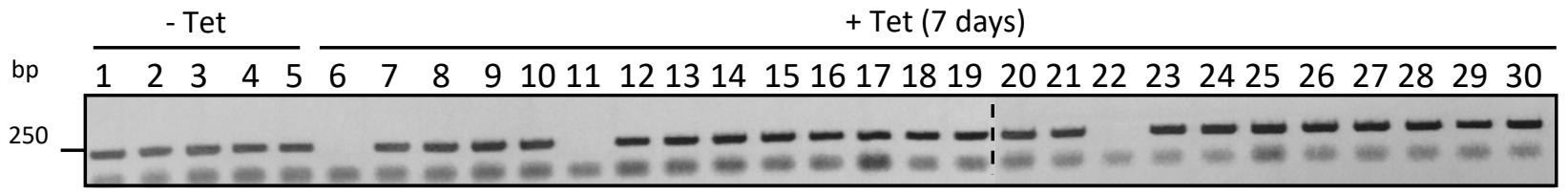

C

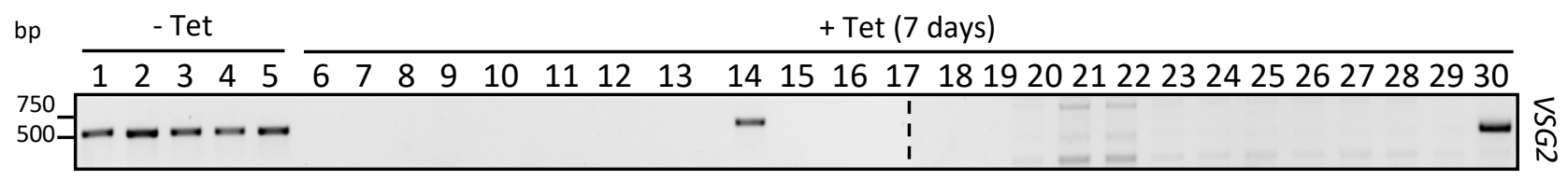


A

B
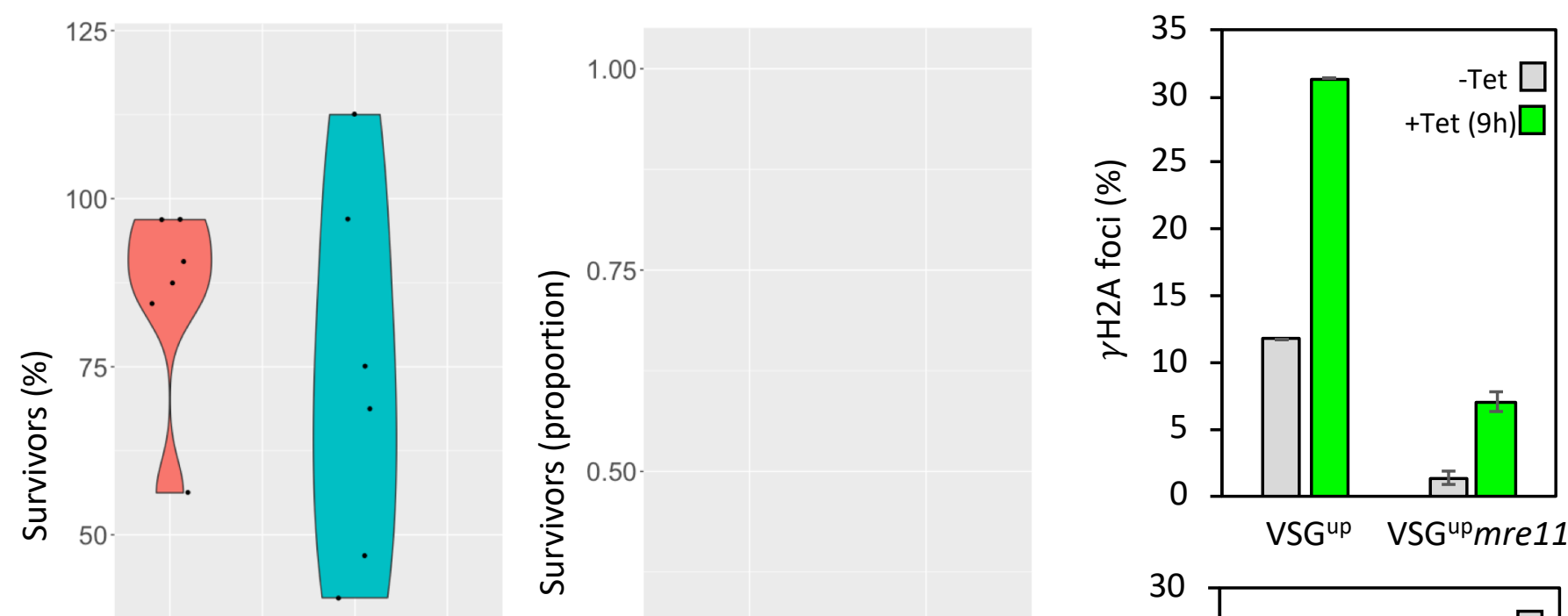

25

0.25

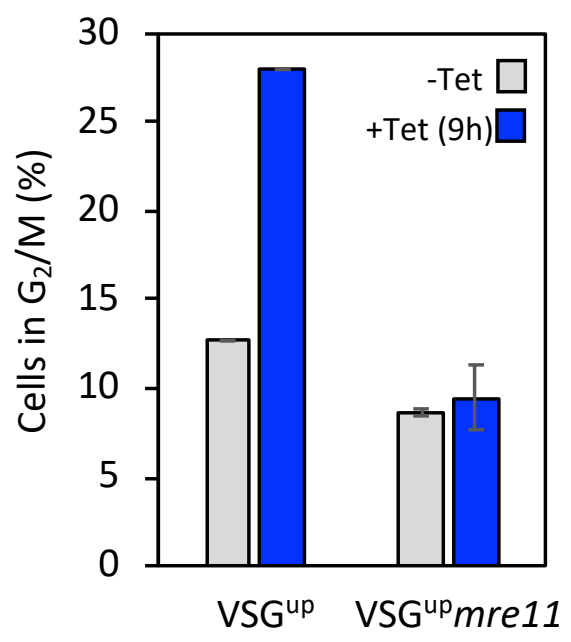

C
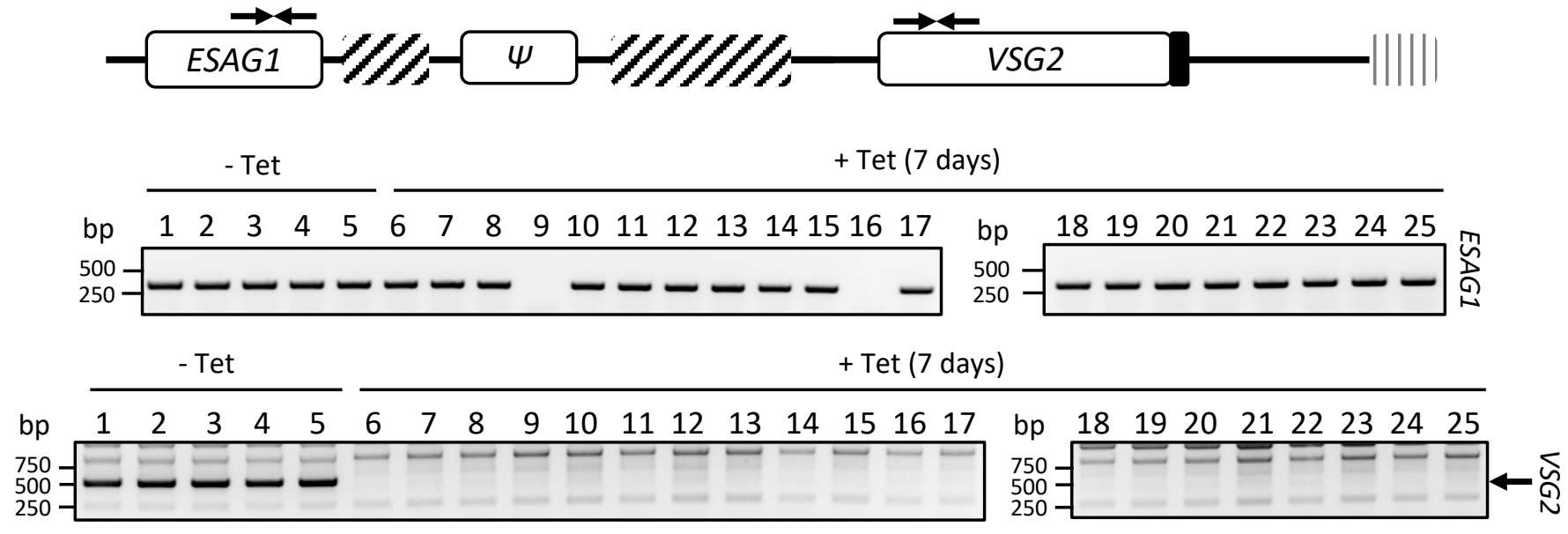


\section{Supplementary Figure 7}

\begin{tabular}{lcc} 
A & & \\
& & \\
& & \\
\hline & VSG & \\
\hline & VSG $^{\text {up }}$ rad50 \\
\hline Total & 83 & 225 \\
\hline BES & 10 & 11 \\
\hline Minichromosome & 22 & 30 \\
\hline Megabase & 29 & 150 \\
\hline Other & 22 & 34 \\
\hline
\end{tabular}

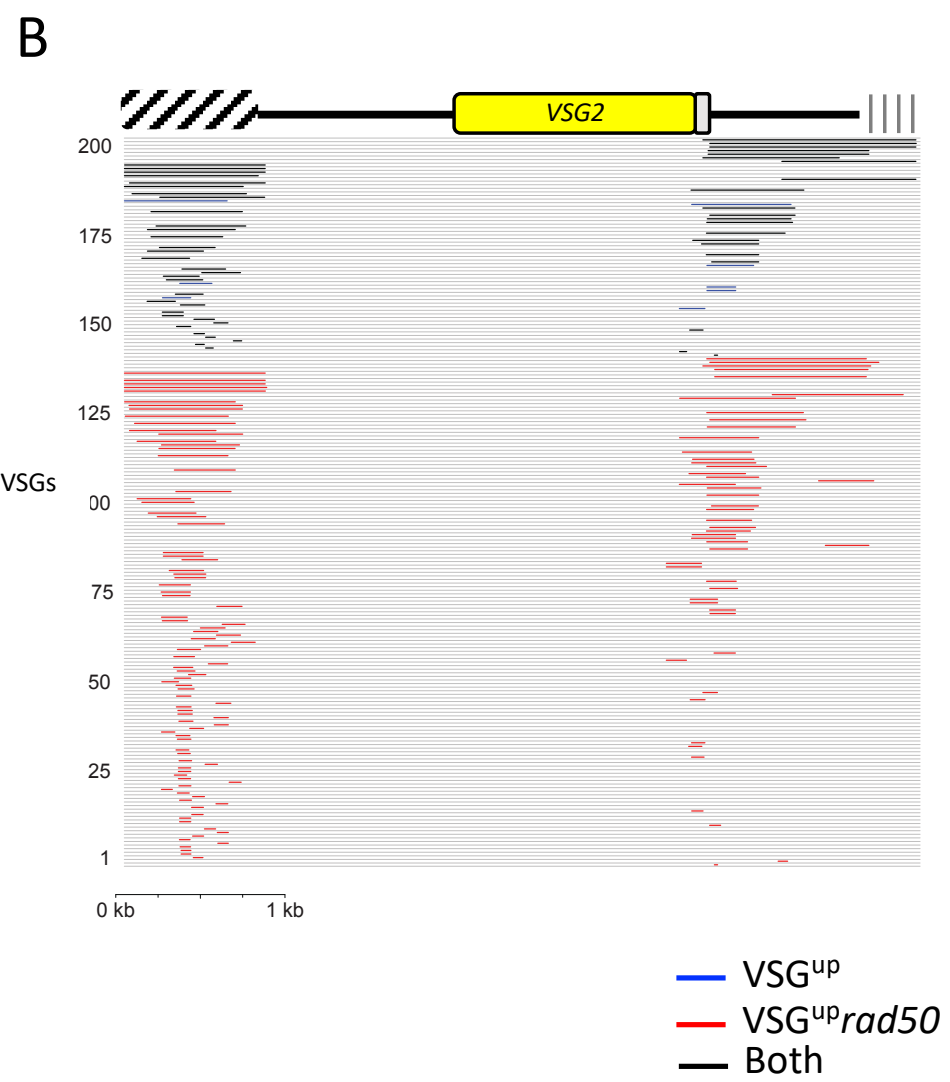




\section{Table 1}

\begin{tabular}{lllllll} 
Strain & $\begin{array}{l}\text { Uninduced } \\
\text { (\%) }\end{array}$ & SD & $\begin{array}{l}\text { Induced } \\
\text { (\%) }\end{array}$ & SD & Proportion SD \\
\hline${ }^{1} \mathrm{HR}$ & 95.09 & $+/-17.1$ & 47.9 & $+/-11.93$ & 0.52 & $+/-0.1$ \\
${ }^{1} \mathrm{HRrad50}$ & 35.76 & $+/-6.83$ & 2.91 & $+/-4.36$ & 0.08 & $+/-0.12$ \\
${ }^{1} \mathrm{HRmre11}$ & 47.27 & $+/-6.97$ & 1.79 & $+/-1.65$ & 0.04 & $+/-0.03$ \\
${ }_{\text {VSG }}^{\text {up }}$ & 85.42 & $+/-15.1$ & 5.45 & $+/-1.81$ & 0.07 & $+/-0.02$ \\
\hline VSG ${ }^{\text {up }}$ rad50 & 51.04 & $+/-16.3$ & 5.81 & $+/-1.64$ & 0.11 & $+/-0.03$ \\
VSG $^{\text {up }}$ mre11 & 73.44 & $+/-27.86$ & 1.75 & $+/-0.37$ & 0.02 & $+/-0.005$
\end{tabular}

